\title{
Discovery of a galaxy overdensity around a powerful, heavily obscured FRII radio galaxy at $z=1.7$ : star formation promoted by large-scale AGN feedback?
}

R. Gilli ${ }^{1}$, M. Mignoli ${ }^{1}$, A. Peca ${ }^{1}$, R. Nanni ${ }^{1,2}$, I. Prandoni ${ }^{3}$, E. Liuzzo ${ }^{3}$, Q. D’Amato ${ }^{2,3}$, M. Brusa ${ }^{2}$, F. Calura ${ }^{1}$, G. B. Caminha ${ }^{4}$, M. Chiaberge ${ }^{5}$, A. Comastri ${ }^{1}$, O. Cucciati ${ }^{1}$, F. Cusano ${ }^{1}$, P. Grandi ${ }^{1}$, R. Decarli ${ }^{1}$, G. Lanzuisi ${ }^{1}$,

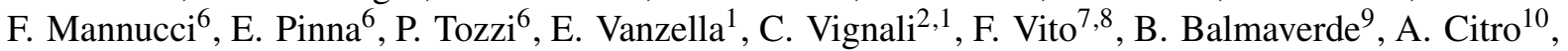
N. Cappelluti ${ }^{11}$, G. Zamorani ${ }^{1}$, and C. Norman ${ }^{5,12}$

${ }^{1}$ INAF - Osservatorio di Astrofisica e Scienza dello Spazio di Bologna, Via P. Gobetti 93/3, 40129 Bologna, Italy e-mail: roberto.gilli@inaf.it

2 Dipartimento di Fisica e Astronomia, Università degli Studi di Bologna, Via P. Gobetti 93/2, 40129 Bologna, Italy

3 INAF - Istituto di Radioastronomia, Via P. Gobetti 101, 40129 Bologna, Italy

${ }^{4}$ Kapteyn Astronomical Institute, University of Groningen, Postbus 800, 9700 Groningen, The Netherlands

5 Space Telescope Science Institute, 3700 San Martin Drive, Baltimore, MD 21218, USA

${ }^{6}$ INAF - Osservatorio Astrofisico di Arcetri, Largo Enrico Fermi 5, 50125 Firenze, Italy

7 Instituto de Astrofisica and Centro de Astroingenieria, Facultad de Fisica, Pontificia Universidad Catolica de Chile, Casilla 306, Santiago 22, Chile

8 Chinese Academy of Sciences South America Center for Astronomy, National Astronomical Observatories, CAS, Beijing 100012, PR China

9 INAF - Osservatorio Astrofisico di Torino, Via Osservatorio 20, 10025 Pino Torinese, Italy

10 Center for Gravitation, Cosmology and Astrophysics, Department of Physics, University of Wisconsin-Milwaukee, 3135 N. Maryland Avenue, Milwaukee, WI 53211, USA

11 Physics Department, University of Miami, Coral Gables, FL 33124, USA

12 Department of Physics and Astronomy, Johns Hopkins University, Baltimore, MD 21218, USA

Received 18 June 2019 / Accepted 27 August 2019

\begin{abstract}
We report the discovery of a galaxy overdensity around a Compton-thick Fanaroff-Riley type II (FRII) radio galaxy at $z=1.7$ in the deep multiband survey around the $z=6.3$ quasi-stellar object (QSO) SDSS J1030+0524. Based on a 6h VLT/MUSE and on a $4 \mathrm{~h} \mathrm{LBT/LUCI}$ observation, we identify at least eight galaxy members in this structure with spectroscopic redshift $z=1.687-1.699$, including the FRII galaxy at $z=1.699$. Most members are distributed within $400 \mathrm{kpc}$ from the FRII core. Nonetheless, the whole structure is likely much more extended, as one of the members was serendipitously found at $\sim 800 \mathrm{kpc}$ projected separation. The classic radio structure of the FRII itself extends for $\sim 600 \mathrm{kpc}$ across the sky. Most of the identified overdensity members are blue, compact galaxies that are actively forming stars at rates of $\sim 8-60 M_{\odot} \mathrm{yr}^{-1}$. For the brightest of them, a half-light radius of $2.2 \pm 0.8 \mathrm{kpc}$ at 8000 Å rest-frame was determined based on adaptive optics-assisted observations with LBT/SOUL in the Ks band. We do not observe any strong galaxy morphological segregation or concentration around the FRII core. This suggests that the structure is far from being virialized and likely constitutes the progenitor of a local massive galaxy group or cluster caught in its main assembly phase. Based on a $500 \mathrm{ks}$ Chandra ACIS-I observation, we found that the FRII nucleus hosts a luminous QSO $\left(L_{2-10 \mathrm{keV}}=1.3 \times 10^{44} \mathrm{erg} \mathrm{s}^{-1}\right.$, intrinsic and rest-frame) that is obscured by Compton-thick absorption $\left(N_{\mathrm{H}}=1.5 \pm 0.6 \times 10^{24} \mathrm{~cm}^{-2}\right)$. Under standard bolometric corrections, the total measured radiative power $\left(L_{\mathrm{rad}} \sim 4 \times 10^{45} \mathrm{erg} \mathrm{s}^{-1}\right)$ is similar to the jet kinetic power that we estimated from radio observations at $150 \mathrm{MHz}\left(P_{\mathrm{kin}}=6.3 \times 10^{45} \mathrm{erg} \mathrm{s}^{-1}\right)$, in agreement with what is observed in powerful jetted AGN. Our Chandra observation is the deepest so far for a distant FRII within a galaxy overdensity. It revealed significant diffuse X-ray emission within the region that is covered by the overdensity. In particular, X-ray emission extending for $\sim 240 \mathrm{kpc}$ is found around the eastern lobe of the FRII. Four out of the six MUSE star-forming galaxies in the overdensity are distributed in an arc-like shape at the edge of this diffuse X-ray emission. These objects are concentrated within $200 \mathrm{kpc}$ in the plane of the sky and within $450 \mathrm{kpc}$ in radial separation. Three of them are even more concentrated and fall within $60 \mathrm{kpc}$ in both transverse and radial distance. The probability of observing four out of the six $z=1.7$ sources by chance at the edge of the diffuse emission is negligible. In addition, these four galaxies have the highest specific star formation rates of the MUSE galaxies in the overdensity and lie above the main sequence of field galaxies of equal stellar mass at $z=1.7$. We propose that the diffuse X-rays originate from an expanding bubble of gas that is shock heated by the FRII jet, and that star formation is promoted by the compression of the cold interstellar medium of the galaxies around the bubble, which may be remarkable evidence of positive AGN feedback on cosmological scales. We emphasize that our conclusions about the feedback are robust because even assuming that the diffuse X-ray emission arises from inverse Compton scattering of photons of the cosmic microwave background by the relativistic electrons in the radio lobe, star formation may be promoted by the nonthermal pressure of the expanding lobe.
\end{abstract}

Key words. galaxies: clusters: general - galaxies: high-redshift - quasars: supermassive black holes - shock waves galaxies: star formation - X-rays: galaxies: clusters 


\section{Introduction}

Distant $(z \gtrsim 1.5)$ protoclusters and large-scale structures are ideal laboratories for investigating the complex processes that led to the assembly of local galaxy clusters. These processes involve mergers and interactions between gas-rich galaxies, fueling and growth of black holes at galaxy centers, and finally the (either positive or negative) active galatic nucleus (AGN) feedback on the intracluster medium (ICM) and on the star formation of member galaxies (see Overzier 2016 for a recent review).

Gas-rich galaxies have been observed in high- $z$ clusters and protoclusters (Hayashi et al. 2017; Noble et al. 2017), with star formation rates up to $800 M_{\odot} \mathrm{yr}^{-1}$ (Santos et al. 2015). A large reservoir of diffuse cold and metal-rich molecular gas $\left(M_{\mathrm{H}_{2}} \sim\right.$ $10^{11} M_{\odot}$ ) extending for $50-70 \mathrm{kpc}$ was found around the radio galaxy at the center of the $z=2.2$ Spiderweb protocluster (Emonts et al. 2018). Its physical properties indicate that this gas is the product of mixing of large-scale gas outflows ejected by supernova ( $\mathrm{SN}$ ) winds and/or AGN activity, and that it is the seed of further star formation (see also Webb et al. 2017 for another example of a large reservoir of molecular gas at the center of a $z=1.7$ cluster). All of the above suggests that the main transition from active to passively evolving galaxies in large-scale structures in fact occurs at $z \sim 1.5-2.0$, which is then a crucial epoch in the formation history of local massive clusters (Overzier 2016).

High- $z$ radio galaxies (HzRGs) are known to be excellent tracers of protoclusters and overdense environments (Pentericci et al. 2000; Miley \& De Breuck 2008; Chiaberge et al. 2010), and several HzRGs were found in which star formation in the host is triggered by the radio jet (Dey et al. 1997; Bicknell et al. 2000). Whether these powerful jets can also trigger star formation in companion galaxies remains an open question, and although numerical simulations indicate this as an efficient mechanism to form stars (Fragile et al. 2017), it has been observed in only a few systems (Croft et al. 2006).

We report here the discovery of a large-scale structure at $z \sim$ 1.7 around a powerful Faranoff-Riley type II (FRII) radio galaxy in the field of the luminous $z=6.3$ quasar SDSS J1030+0524. The presence of a powerful radio galaxy was revealed in 2003 by means of an observation of the quasar field at $1.4 \mathrm{GHz}$ with the Very Large Array (VLA; Petric et al. 2003). Nanni et al. (2018) reanalyzed these data and measured the flux, morphology, and extension of the radio galaxy. The object displays a classic FRII morphology, with an unresolved core (at 1.5" angular resolution), a jet pointing eastward, and two bright lobes (the west lobe is $>6$ times brighter than the east lobe), extending for 1.2 arcmin in total. In this paper we present the spectroscopic redshift measurement of the FRII host and the discovery of nearby galaxies that form a large-scale structure around it, as well as Chandra $\mathrm{X}$-ray observations of the whole structure.

The paper is structured as follows: In Sect. 2 we present the entire set of multiband data that are available to study the galaxy overdensity. In Sect. 3 we describe the reduction and analysis of the data obtained (i) at the Large Binocular Telescope (LBT) with the LBT Utility Camera in the Infrared (LUCI) and the Single conjugate adaptive Optics Upgrade for LBT (SOUL), (ii) with the Multi Unit Spectroscopic Explorer (MUSE) at the Very Large Telescope (VLT), and (iii) with the $2 \times 2$ array of the Advanced CCD Imaging Spectrometer (ACIS-I) on board the Chandra X-ray Observatory. In Sect. 4 we present the results on the structure of the overdensity, star formation of its member galaxies, and power and obscuration of the FRII nucleus. In Sect. 5 we discuss the total mass of the overdensity, the presence of another radio source that is a candidate member, the origin and interpretation of the diffuse X-ray emission, and finally, the evidence of positive AGN feedback on the star formation of some overdensity members. Our conclusions are presented in Sect. 6.

A concordance cosmology with $H_{0}=70 \mathrm{~km} \mathrm{~s}^{-1} \mathrm{Mpc}^{-1}$, $\Omega_{\mathrm{m}}=0.3$, and $\Omega_{\Lambda}=0.7$, in agreement within the errors with the Planck 2015 results (Planck Collaboration XIII 2016), and a Salpeter (1955) initial mass function (IMF) are used throughout this paper. In the adopted cosmology, the angular scale at $z=1.7$ is $8.5 \mathrm{kpc} \operatorname{arcsec}^{-1}$.

\section{Multiband survey in the $\mathbf{J 1 0 3 0}$ field}

We have accumulated a rich dataset of deep-and-wide multiband observations in the field centered at $\mathrm{RA}=10^{\mathrm{h}} 30^{\mathrm{m}} 27^{\mathrm{s}}$ Dec $=+05^{\circ} 24^{\prime} 55^{\prime \prime}$ (hereafter the J1030 field), as a result of the intensive follow-up of the $z=6.3$ quasar SDSS J1030+0524, the first discovered at $z>6$ (Fan et al. 2001), and of its small-to-large scale environment, which features the best candidate overdensity of galaxies around a quasar at such redshifts (Stiavelli et al. 2005; Kim et al. 2009; Morselli et al. 2014; Balmaverde et al. 2017). Our team is leading a major observational effort in the J1030 field by collecting and reanalyzing multiband data from major international facilities. Morselli et al. (2014) presented optical imaging in the $r, i, z$ filters obtained at the LBT using the Large Binocular Camera (LBC). Balmaverde et al. (2017) presented nearIR imaging in the $Y$ and $J$ filters obtained at the Canada France Hawaii Telescope using WIRCam (CFHT/WIRCam). Representative limiting magnitudes $(5 \sigma, \mathrm{AB})$ of $27.5,25,24$ in $r, z, J$, respectively, were obtained in these observations.

The field is part of the Multiwavelength Yale-Chile survey (MUSYC; Gawiser et al. 2006), which provides additional imaging in $U B V R I z J H K$ down to $B=26$ and $K=23 \mathrm{AB}$, for instance (see also Quadri et al. 2007), and has also been entirely observed by Spitzer IRAC down to $24.5 \mathrm{AB}$ mag at $3.6 \mu \mathrm{m}$, for example ( $3 \sigma$; Annunziatella et al. 2018). In 2017 we observed the field with Chandra ACIS-I for $500 \mathrm{ks}$ (Nanni et al. 2018), making this field the fourth deepest extragalactic X-ray survey to date, and at the same time, the deepest X-ray observation of both a $z \sim 6$ QSO and of a distant galaxy overdensity around a powerful FRII galaxy.

The central part of the field has also been observed with the Hubble Space Telescope (HST) Advanced Camera for Surveys (ACS; Stiavelli et al. 2005; Kim et al. 2009) and Wide Field Camera 3 (WCF3; PI Simcoe, unpublished), and VLT/MUSE (see Sect. 3.2). The host of the FRII radio galaxy falls at only 40 arcsec southwest of the QSO (see Fig. 1), and together with the inner regions of the overdensity, has then been covered by most imaging data in the field.

Optical and near-IR spectroscopic follow-up of the sources in the field is being conducted through dedicated campaigns at the LBT (with both the multi-object optical spectrograph MODS and the near-IR spectrograph LUCI), Keck (DEIMOS), and VLT (FORS2) telescopes. Detailed information about the full multiband imaging and spectroscopic coverage of the J1030 field can be found at the survey website ${ }^{1}$.

\section{Data reduction and analysis}

\section{1. $L B T / L U C I$}

The LBT/LUCI near-IR spectrum of the FRII radio galaxy at $\mathrm{RA}=10^{\mathrm{h}} 30^{\mathrm{m}} 25.2 \mathrm{Dec}=+05^{\circ} 24^{\prime} 28^{\prime \prime}$ was obtained as part of the INAF-LBT Strategic Program ID 2017/2018 \#18 (P.I. R. Gilli).

\footnotetext{
http: //www . oabo.inaf.it/ LBTz6/1030
} 


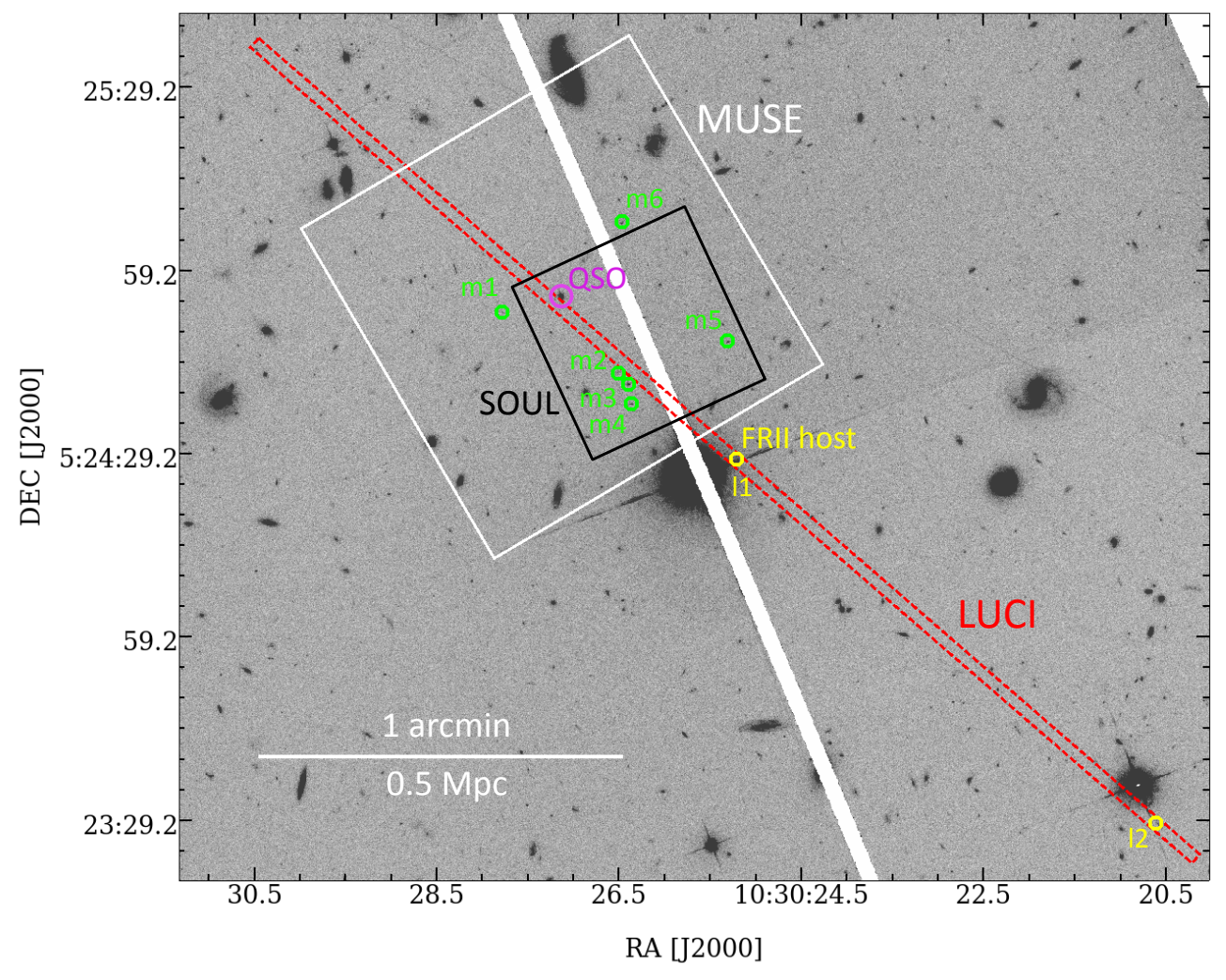

Fig. 1. HST/ACS F850LP image of the J1030 field (north is up and east is to the left). The white strip running across a very bright star is the gap between the two ACS CCDs. The positions of the LUCI long-slit $\left(1^{\prime \prime} \times 205^{\prime \prime}\right)$ observation of the FRII host and of the $60^{\prime \prime} \times 60^{\prime \prime}$ MUSE pointing are shown as a dashed red rectangle and a white square, respectively. The $30^{\prime \prime} \times 30^{\prime \prime}$ region observed with SOUL is shown as a black square. The yellow circles mark the FRII host at $z=1.699$ (l1) and a star-forming galaxy at $z=1.697(l 2)$ that was found serendipitously along the LUCI slit. MUSE galaxies in the redshift range [1.687-1.697] are also shown as green circles and labeled $(m 1-m 6)$. The position of the $z=6.3$ QSO SDSS J1030+0524 is also marked in magenta.
This is a large observing project devoted to spectroscopically follow up X-ray and radio sources in the J1030 field. The position of the FRII host in the J1030 field is shown in Fig. 1. It falls close to a very bright star, but it is relatively bright in the near-IR bands, and can therefore be accurately placed within a slit.

The FRII radio galaxy was observed during the nights of 2018 February 4 and 5 in the HKspec (1.47-2.35 $\mu \mathrm{m}$ ) band using the G200 grating. The total integration time was $4 \mathrm{~h}$, achieved through 72 individual exposures of $200 \mathrm{~s}$ each, and the single exposures were dithered along the slit. A slit of $1.0 \operatorname{arcsec} \times$ 3.4 arcmin was used. The spectral resolution is $R \approx 2000$. The LUCI data were reduced by the INAF LBT Spectroscopic Reduction Center in Milan ${ }^{2}$. The LBT spectroscopic pipeline was developed following the VIMOS experience (Garilli et al. 2012), but the reduction of LUCI spectroscopic data includes new dedicated sky subtraction algorithms.

The analysis of the reduced 2D and 1D spectra of the radio galaxy is highlighted by the clear presence of a bright emission line at $\lambda=1.771 \mu \mathrm{m}$ (see Fig. 2, top panel). The spectral feature is a clear blend of two resolved emission lines, with an intrinsic FWHM of $\approx 600 \mathrm{~km} \mathrm{~s}^{-1}$. The observed FWHM was deconvolved by subtracting in quadrature the instrumental resolution as determined from adjacent sky lines. We identified the emission lines with the $\mathrm{H} \alpha$ and [NII] $6583 \AA$ complex, and to measure the redshift, we performed a multiple fit with three Gaussians. The relative intensity of the two lines of the [NII] doublet was fixed to the value of 3 (Acker et al. 1989), and the width of the [NII] lines was matched to that of $\mathrm{H} \alpha$. The continuum was fit with a linear function in two spectral windows adjacent to the blended emission. No broad Balmer component is required by the fit, but the relatively low signal-to-noise ratio $(\mathrm{S} / \mathrm{N})$ of the spectrum precludes any definitive conclusion regarding the presence of an additional shallow broad component. The redshift obtained by

\footnotetext{
2 http://www.iasf-milano.inaf.it/software
}

the fit is $1.6987 \pm 0.0002$. The measured flux and luminosity of the $\mathrm{H} \alpha$ line are $2.2 \times 10^{-16} \mathrm{erg} \mathrm{cm}^{-2} \mathrm{~s}^{-1}$ and $4.4 \times 10^{42} \mathrm{erg} \mathrm{s}^{-1}$ (with a $\sim 14 \%$ error), respectively. The measured value of the $[\mathrm{NII}] 6583 / \mathrm{H} \alpha$ ratio is $\sim 0.6-0.7$, indicating, together with the line FWHMs, that the FRII radio galaxy hosts an obscured (type 2) AGN (Cid Fernandes et al. 2011).

Within the LUCI slit, at a distance of 1.5 arcmin southwest from the radio source (see Fig. 1), a serendipitous galaxy shows a spectrum with an unresolved emission line at $1.7695 \mu \mathrm{m}$. We identified the line as $\mathrm{H} \alpha$ at $z=1.6966$. This redshift measurement is also supported by the detection of a faint [NII]6583 emission feature. The measured flux and luminosity of the $\mathrm{H} \alpha$ line are $2.4 \times 10^{-17} \mathrm{erg} \mathrm{cm}^{-2} \mathrm{~s}^{-1}$ and $4.8 \times 10^{41} \mathrm{erg} \mathrm{s}^{-1}$ (with a $\sim 25 \%$ error), respectively. The narrowness of the $\mathrm{H} \alpha$ emission line, the low $[\mathrm{NII}] / \mathrm{H} \alpha$ flux ratio $(<0.2)$, and the blue color of the galaxy are all suggestive of a young star-forming galaxy. Its near-IR spectrum is shown in Fig. 2 (bottom panel).

\subsection{VLT/MUSE}

The field of SDSS J1030+0524 was observed by MUSE (Bacon et al. 2010, 2014) between June and July 2016 under the program ID 095.A-0714 (PI Karman) for a total of $\approx 6.4 \mathrm{~h}$ of exposure time. The observations consist of a total of 16 target exposures with a small dither pattern and $90^{\circ}$ rotations to reduce instrumental features in the final stacked data. We used the MUSE reduction pipeline version 1.6.2 (Weilbacher et al. 2014) to process the individual exposures and to create the final stacked data cube. All usual calibration recipes (bias, flat field, wavelength, flux calibration, etc.) were applied to the raw exposures in order to create the corresponding data cubes and PIXELTABLEs. We checked each single data cube but failed to find large differences between them. The final data cube (with the full $6.4 \mathrm{~h}$ depth) was created by combining the reduced PIXELTABLEs taking into account the small offsets between exposures. In a 


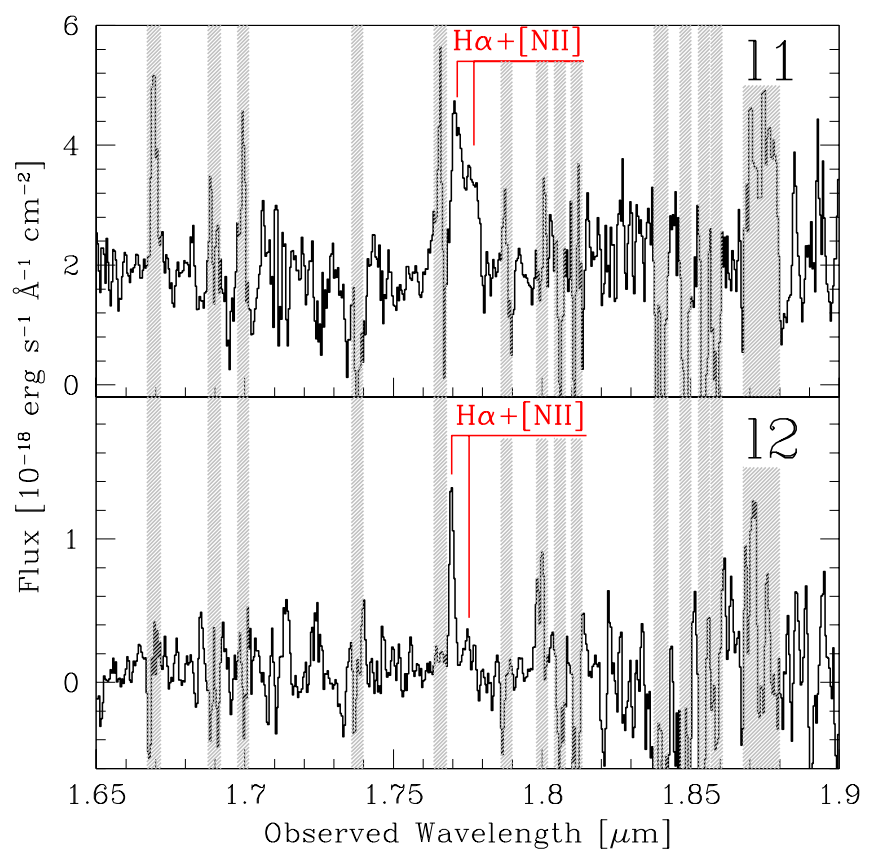

Fig. 2. Top: LBT/LUCI near-IR spectrum of the FRII host at $z=1.6987$. Bottom: LBT/LUCI near-IR spectrum of the serendipitous galaxy found at $z=1.6966$. H $\alpha$ and [NII] are marked in both panels. Gray bands show spectral regions with strong sky lines.

final step we defined the sky regions from the white image and applied the Zurich atmosphere purge (ZAP version 1.0, Soto et al. 2016) to reduce sky residuals that were still present on the data. The final data cube has a wavelength range of $4750 \AA-$ $9350 \AA$, a spectral sampling of $1.25 \AA$ and a spatial sampling of $0^{\prime \prime} .2$, covering an area of 1 sq-arcmin (see Fig. 1) centered at $\mathrm{RA}=10^{\mathrm{h}} 30^{\mathrm{m}} 27^{\mathrm{s}} \mathrm{Dec}=+05^{\circ} 24^{\prime} 55^{\prime \prime}$, that is, the sky position of the QSO SDSSJ1030+0524. The seeing conditions were very good, with an average seeing of 0.6 arcsec, as directly measured on bright stars in the MUSE data cube.

To identify the sources in the MUSE field of view (FoV), we used SExtractor (Bertin \& Arnouts 1996) on the white image, obtained by summing the flux at all wavelengths for each spaxel. To avoid the loss of objects with extreme colors, we also ran SExtractor on sliced images in three different wavelength ranges (each $1500 \AA$ wide). After combining the four lists and performing a visual inspection to remove artifacts, we had a sample of 138 sources in the MUSE data cube, including the central quasi-stellar object (QSO). For each source we extracted a 1D spectrum by combining the spaxels inside a 3-pixel aperture that matches the seeing FWHM. We successfully measured the redshift up to $z \gtrsim 6$ for 87 of the 138 sources. As expected for such a small sky region, the redshift distribution is spiky, but a particularly striking feature is present at $z \sim 1.7$, which is where the FRII host and the serendipitous source in the LUCI slit also lie (see Fig. 3), with 5(6) MUSE galaxies within $\Delta z<$ $0.0018(0.096)$, that is, within 200(1060) $\mathrm{km} \mathrm{s}^{-1}$ (see also Fig. 4). This is noteworthy because measuring redshifts with MUSE is particularly difficult at this redshift owing to the absence of strong emission lines in the spectral range. For the two brightest galaxies ( $m 3$ and $m 4$ ), the redshifts were measured by finding seven interstellar absorption lines (produced by FeII and MgII transitions), computing redshifts for each identified line, and combining them into a single average value. For the other galaxies the redshift was obtained by cross-correlating their spectra

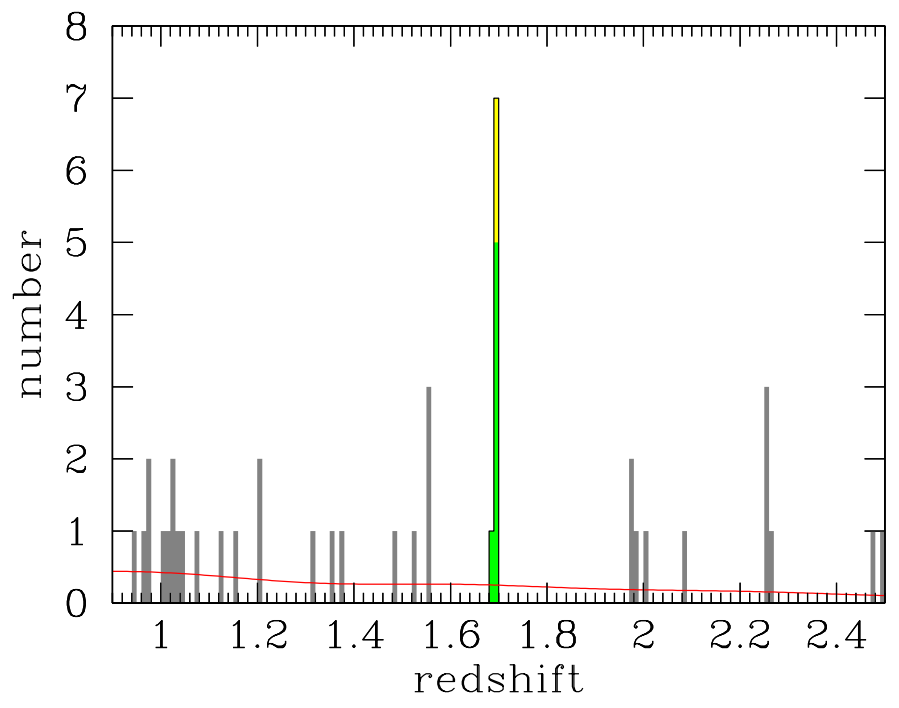

Fig. 3. Redshift distribution of MUSE sources at $z=0.9-2.5$ in bins of $\Delta z=0.01$ (gray histogram). The six MUSE sources $(m 1-m 6)$ in the $z=1.69$ overdensity are shown in light green. The red curve shows the expected background curve, obtained by smoothing the MUSE redshift distribution, used to quantify the significance of the redshift structure. The two additional sources at $z=1.69$ found by LUCI (including the FRII host) are shown in yellow.

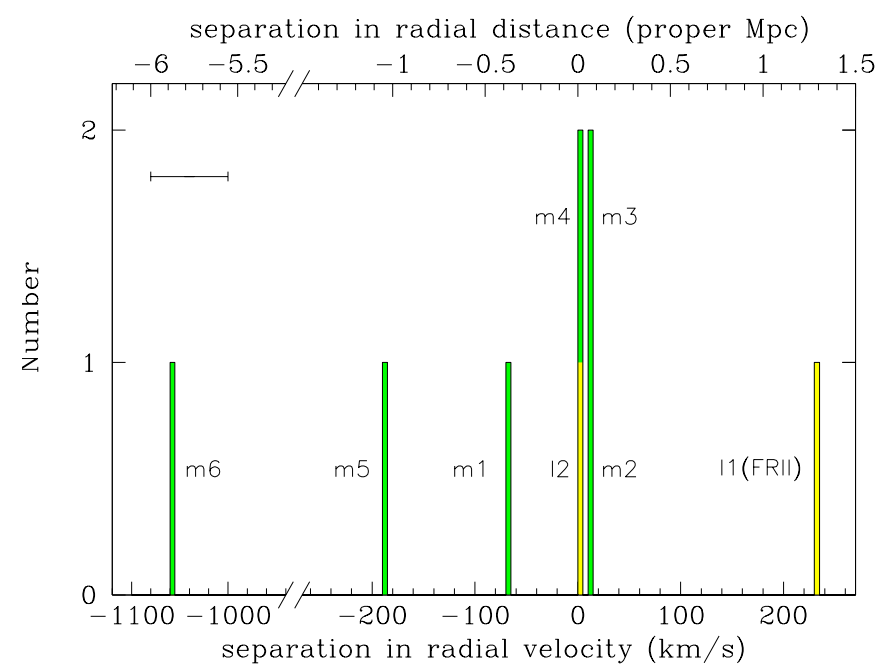

Fig. 4. Distribution of the eight overdensity members in rest-frame radial velocity space (lower $x$-axis) and in radial separations (upper $x$ axis), assuming the median redshift of the sample as the zero point. Radial separations are computed assuming that the overdensity members have negligible peculiar velocities. Velocity bins are $5 \mathrm{~km} \mathrm{~s}^{-1}$ wide. Green and yellow bars refer to redshifts measured by MUSE and LUCI, respectively, and the position of the FRII host is also labeled. The four MUSE sources in the arc around the diffuse X-ray emission $(m 1-m 4)$ are all concentrated within $450 \mathrm{kpc}$ radial, and the three of them that lie within $60 \mathrm{kpc}$ on the plane of the sky $(m 2-m 4)$, also lie within $60 \mathrm{kpc}$ in the radial direction. The typical uncertainty introduced by redshift errors $(\Delta z \pm 0.0004$, see Table 1$)$ is shown by the horizontal bar.

with a star-forming template. Interstellar UV absorption lines are known to have velocity offsets with respect to the nebular emission lines that trace the galaxy systemic velocities. To correct MUSE redshifts to the systemic redshifts and to make them comparable with the redshifts of LUCI spectra, we applied a correction for interstellar gas outflows of $\Delta v=135 \pm 22 \mathrm{~km} \mathrm{~s}^{-1}$ (from Talia et al. 2012). In all of them the redshift is confirmed by 


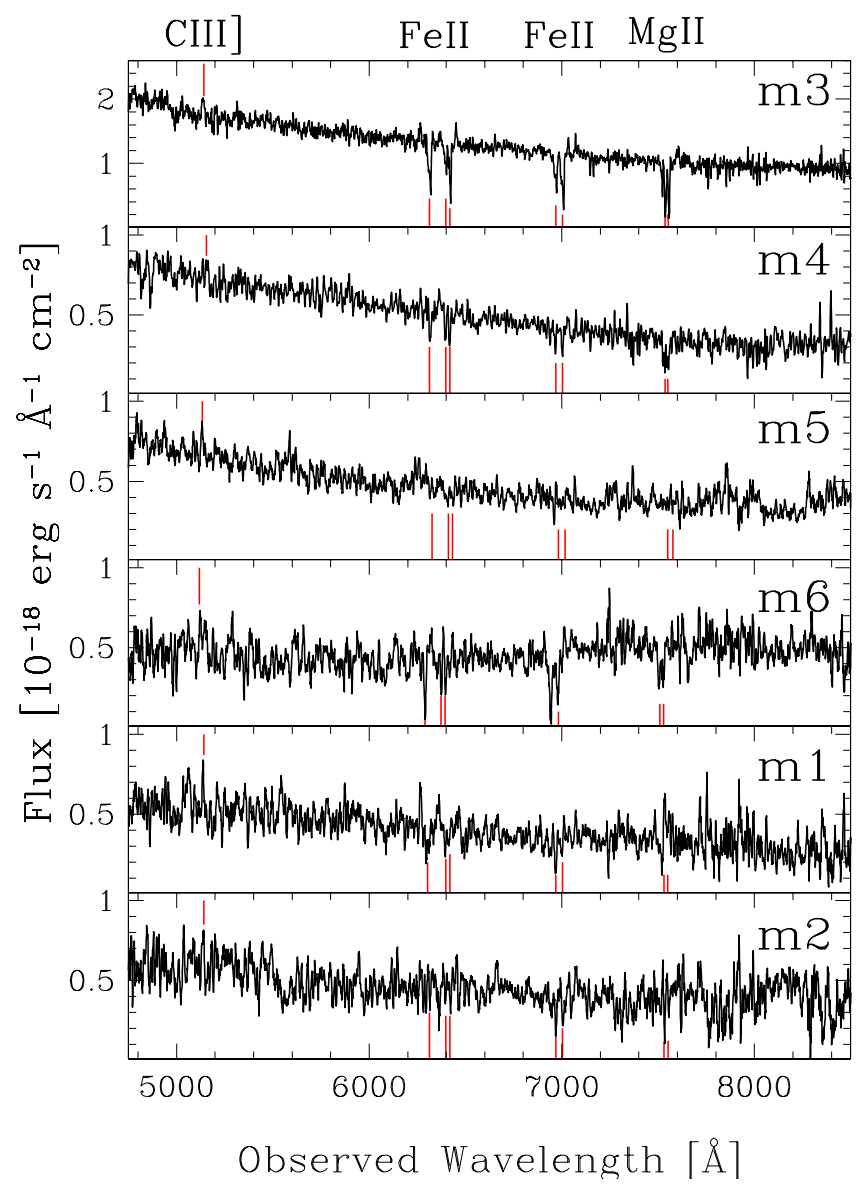

Fig. 5. MUSE spectra of the six star-forming galaxies in the $z=1.7$ overdensity sorted by decreasing UV flux. The main absorption and emission lines used in the redshift determination are labeled.

the detection of the faint CIII] $\lambda 1909$ emission line. The MUSE spectra of the six galaxies in the $z=1.7$ spike are shown in Fig. 5.

\subsection{LBT adaptive-optics observations with SOUL}

The J1030 field was observed using the new adaptive-optics (AO) system SOUL on the LBT (Pinna et al. 2016). This is an upgrade of the existing AO system (FLAO; Esposito et al. 2011) implementing a pyramid wavefront sensor for a natural guide star (NGS) and an adaptive secondary mirror. With respect to FLAO, SOUL allows for a better AO correction and the use of fainter NGS. Observations were obtained on 8 April 2019 during the commissioning of the SOUL system with the LUCI1 near-IR camera. The bright star $(R \sim 12)$ close to the FRII radio galaxy (see Fig. 1) allows for high-resolution AO observations. The field was observed for $40 \mathrm{~min}$ in the $K s$ filter under seeing between $0.8^{\prime \prime}$ and $1.0^{\prime \prime}$ FWHM, and the data were reduced with standard procedures. Although the reference star was found to be a double system with $0.4^{\prime \prime}$ of separation and a factor of $\sim 4$ in flux ratio, the $\mathrm{AO}$ correction provided a point spread function (PSF) down to $F W H M=72 \times 76$ mas at $23^{\prime \prime}$ of distance to the NGS on the single one-minute images. The final combined image has $F W H M=90 \times 120$ mas at the same distance.

One of the MUSE galaxies in the structure at $z=1.7(\mathrm{~m} 3$, see Fig. 1 and Table 1) and a radio galaxy that is also a candidate source at the same redshift (see Sect. 5.2) were detected with high S/N (see Fig. 6). The two galaxies are well resolved, but the depth of the image is not enough to perform a full PSF deconvolution and morphological fitting. Nevertheless, a reliable halflight radius $r_{50}$ can be measured by aperture photometry (circular apertures were used). For $m 3$ we obtained $r_{50}=0.26 \pm 0.1^{\prime \prime}$, corresponding to $2.2 \pm 0.8 \mathrm{kpc}$ at $z \sim 1.7$, and for the radio galaxy we obtained $r_{50}=0.27 \pm 0.05^{\prime \prime}$, corresponding to $2.3 \pm 0.4 \mathrm{kpc}$ at $z \sim 1.7$

\subsection{Chandra/ACIS-I}

We observed the J1030 field with Chandra/ACIS-I for a total of $479 \mathrm{ks}$. The observation was divided into ten different pointings with roughly the same aim point performed between January and May 2017. The data were taken in the VFAINT mode, processed using CIAO v4.8, and filtered using standard ASCA grades. The astrometry of each pointing was registered to a reference source catalog that we derived from CFHT/WIRCam observations in the $Y$ and $J$ bands (Balmaverde et al. 2017). More details on the Chandra data reduction are given in Nanni et al. (2018). An Xray source catalog is being derived from these observations that is based on CIAO WAVDETECT (Freeman et al. 2002) for source detection and on ACIS Extract (Broos et al. 2010) for source photometry (Nanni et al., in prep.).

\subsubsection{FRII nucleus}

The core of the FRII corresponds to the X-ray source XID189 in the catalog above. XID189 is only detected in the hard $2-7 \mathrm{keV}$ band $(>5 \sigma)$ with a flux of $f_{2-7}=2.2_{-0.4}^{+0.3} \times 10^{-15} \mathrm{erg} \mathrm{cm}^{-2} \mathrm{~s}^{-1}$. We extracted the spectra of XID189 from each individual pointing and combined them using COMBINE_SPECTRA in CIAO. A circle of $1.5^{\prime \prime}$ radius was used as the source extraction region. A similar procedure was used to derive the background spectrum using an annulus around the source with inner and outer radius of $3^{\prime \prime}$ and 6", respectively. The X-ray spectrum of XID189 was grouped to a minimum of one count per energy bin and then analyzed with XSPEC v12.5.3 using the $C$-statistic (Cash 1979) to estimate the best-fit parameters. All errors are given at the $1 \sigma$ level. A total of $31_{-6}^{+7}$ net counts were measured in the $0.5-$ $7 \mathrm{keV}$ range (1.3-19 keV rest-frame). We fit the spectrum using a simple absorbed power-law model. The absorption was modeled through XSPEC PLCABS (Yaqoob 1997), which assumes an isotropic source of photons enshrouded in a spherical matter distribution. The advantage of this model with respect to the commonly used ZPHABS or ZWABS is that in addition to photoelectric absorption, it correctly accounts for the effects of Compton scattering (at least up to column densities of $5 \times 10^{24} \mathrm{~cm}^{-2}$ and rest-frame energies of $\sim 20 \mathrm{keV}$ ). The use of more sophisticated absorption models such as MYTORUS (Murphy \& Yaqoob 2009) would be inadequate for the low photon statistics measured in XID189. When we fixed the power-law photon index to $\Gamma=1.8$, we measured a column density of $N_{\mathrm{H}}=1.5_{-0.5}^{+0.6} \times 10^{24} \mathrm{~cm}^{-2}$ and absorption-corrected luminosity of $L_{X}=1.3 \times 10^{44} \mathrm{erg} \mathrm{s}^{-1}$ in the $2-10 \mathrm{keV}$ band rest-frame, which qualify XID189 as a heavily obscured, Compton-thick quasar. The Chandra image and spectrum of XID189 are shown in Fig. 7.

\subsubsection{Diffuse X-ray emission}

Our deep Chandra observation revealed several regions of significant diffuse X-ray emission within the area covered by the galaxy overdensity. In Fig. 8 (left) we show a map of the extended X-ray emission obtained by first removing pointlike X-ray sources from the Chandra $0.5-7 \mathrm{keV}$ image, hence 
Table 1. Spectroscopically confirmed overdensity members.

\begin{tabular}{lccccc}
\hline \hline ID & RA & Dec & $K_{\mathrm{AB}}$ & $z_{\mathrm{spec}}$ & Notes \\
$(1)$ & $(2)$ & $(3)$ & $(4)$ & $(5)$ & $\begin{array}{c}\text { arc } \\
\text { (6) }\end{array}$ \\
\hline$m 1$ & $10: 30: 27.73$ & $+05: 24: 52.3$ & $23.97 \pm 0.45$ & $1.6960 \pm 0.0005$ & $\operatorname{arc}$ \\
$m 2$ & $10: 30: 26.46$ & $+05: 24: 42.4$ & $23.14 \pm 0.23$ & $1.6967 \pm 0.0004$ & $\operatorname{arc}$ \\
$m 3$ & $10: 30: 26.34$ & $+05: 24: 40.5$ & $22.21 \pm 0.14$ & $1.6967 \pm 0.0002$ & $\operatorname{arc}$ \\
$m 4$ & $10: 30: 26.31$ & $+05: 24: 37.4$ & $22.67 \pm 0.28$ & $1.6966 \pm 0.0003$ & $\operatorname{arc}$ \\
$m 5$ & $10: 30: 25.26$ & $+05: 24: 47.6$ & $23.34 \pm 0.34$ & $1.6949 \pm 0.0004$ & - \\
$m 6$ & $10: 30: 26.42$ & $+05: 25: 07.1$ & $22.12 \pm 0.21$ & $1.6871 \pm 0.0003$ & - \\
$l 1$ & $10: 30: 25.20$ & $+05: 24: 28.4$ & $20.90 \pm 0.12$ & $1.6987 \pm 0.0002$ & FRII \\
$l 2$ & $10: 30: 20.56$ & $+05: 23: 28.7$ & $22.92 \pm 0.30$ & $1.6966 \pm 0.0004$ & - \\
\hline
\end{tabular}

Notes. Columns: (1) Source+redshift identifier: $m *$ and $l *$ refer to measurements by MUSE and LUCI, respectively; (2) and (3) source coordinates from either MUSE or LBT/LBC $z$-band data; (4) $K$-band magnitude from the MUSYC-deep catalog (Quadri et al. 2007). For $m 1, l 1$ and $l 2$ we manually performed $K$-band photometry on the MUSYC-deep image. (5) Redshift and $1 \sigma$ error; (6) Notes: Galaxies distributed in the arc-like shape at the edge of component A of the diffuse X-ray emission (see text) are labeled "arc".
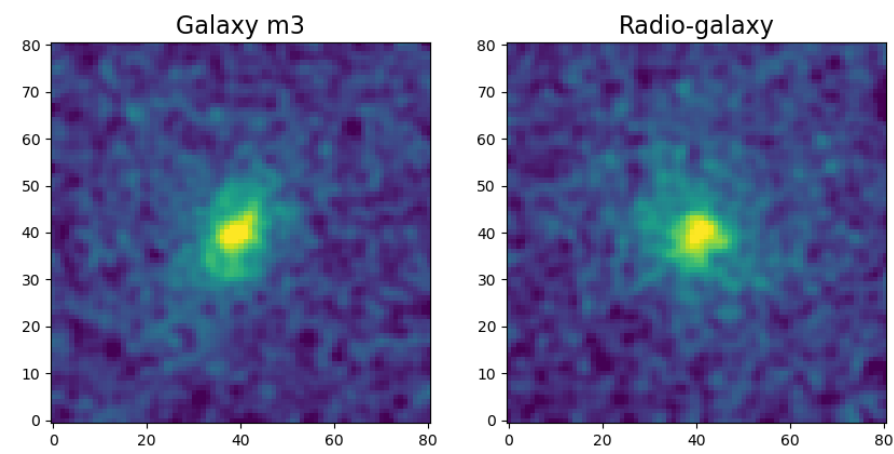

Fig. 6. $K s$-band images of the galaxy $m 3$ (left) and of the radio galaxy that is the candidate overdensity member discussed in Sect. 5.2 (right) obtained with the AO system SOUL at the LBT. Each cutout is $1.2^{\prime \prime} \times$ $1.2^{\prime \prime}$, and the axes are in pixel units (the scale is $15 \mathrm{mas} \mathrm{pixel}^{-1}$ ).

replenishing the "holes" in the image with photons extracted from local background regions (using the CIAO DMFILTH tool), and finally smoothing it with the CSMOOTH tool using smoothing scales up to 10 pixels $\left(\sim 5^{\prime \prime}\right)$. We remark that all measurements presented in the following were performed on the unsmoothed images. The background was evaluated in a rectangular sourcefree region of equal and uniform exposure north of the diffuse emission. Spectral analysis was performed with XSPEC v12.5.3 on spectra grouped to at least one count per bin and using the $C$-statistic. At least three spots of significant $\mathrm{X}$-ray emission are seen, which are labeled A, B, and C in Fig. 8 (left). The most prominent emission (component A) is detected with $S / N=5.5$ in the $0.5-2 \mathrm{keV}$ band. It extends for $\sim 30^{\prime \prime} \times 20^{\prime \prime}$ and overlaps with the eastern lobe of the FRII galaxy. Significant emission $(S / N \sim 5)$ is also found along the direction of the radio jet (component B in Fig. 8 left). Finally, low-significance emission ( $S / N \sim 2.4$ and 3.4 in the full and soft band, respectively) is found at 20 arcsec northeast of the FRII western lobe (component $\mathrm{C}$ in Fig. 8 left). Despite its low significance, we consider component $\mathrm{C}$ as real because it is also visible in the $\mathrm{X}$-ray images that have independently been obtained with XMM-Newton (see, e.g., Nanni et al. 2018).

We note that the significant detection of diffuse X-ray emission around such a distant FRII galaxy has been made possible by the exceptionally deep Chandra observation of the system. We inspected our Chandra data by cutting the exposure at $120 \mathrm{ks}$ and $200 \mathrm{ks}$, performed aperture photometry of

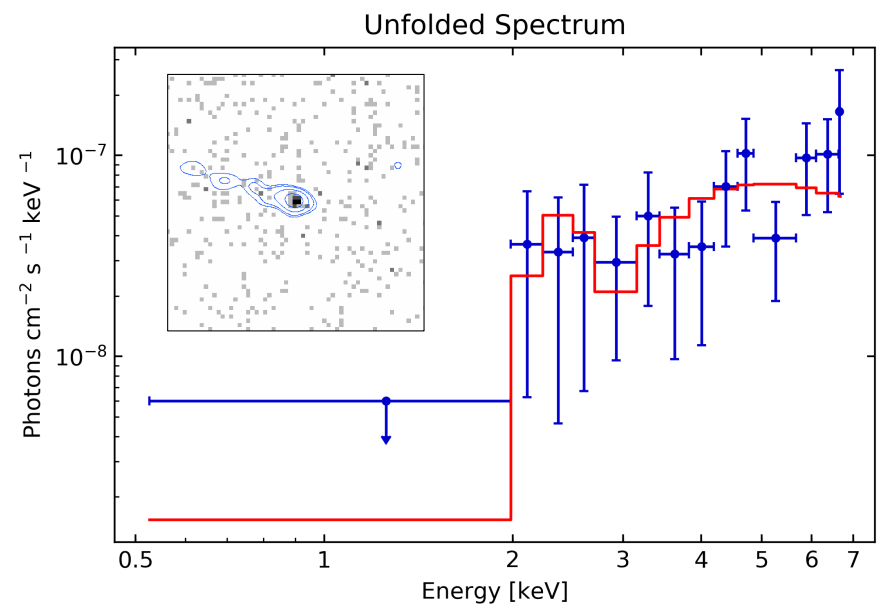

Fig. 7. Response-corrected Chandra/ACIS-I X-ray spectrum of the FRII nucleus (XID189) and best-fit model (in red). By fixing the photon index to 1.8 , a best-fit column density of $N_{\mathrm{H}}=1.5_{-0.5}^{+0.6} \times 10^{24} \mathrm{~cm}^{-2}$ and an intrinsic deabsorbed luminosity in the $2-10 \mathrm{keV}$ rest-frame of $L_{\mathrm{X}}=1.3 \times 10^{44} \mathrm{erg} \mathrm{s}^{-1}$ are obtained, which qualify XID189 as a Compton-thick QSO. The spectrum has been rebinned for display purposes. The inset shows a $30^{\prime \prime} \times 30^{\prime \prime}$ cutout of the $0.5-7 \mathrm{keV}$ Chandra raw image around the source. VLA radio contours at $1.4 \mathrm{GHz}$ are overlaid in blue (with a $\sqrt{3}$ geometric progression starting at $30 \mu \mathrm{Jy}^{\text {beam }}{ }^{-1}$ ).

component $\mathrm{A}$, and compared the results with those from the final exposure. Again, in the unsmoothed data, the $\mathrm{S} / \mathrm{N}$ of component $\mathrm{A}$ in the $0.5-7 \mathrm{keV}$ band increases from 2.1 to 3.3 and 5.5 with increasing exposure. Even considering the brightest of the diffuse X-ray components, this can therefore be detected significantly $(S / N>3)$ only with Chandra exposures larger than $\sim 200 \mathrm{ks}$.

We further investigated the diffuse X-ray emission in the soft and hard band separately, by smoothing point-like subtracted X-ray images following the procedure described above. In Fig. 9 we show the overlap between the soft and hard diffuse X-ray emission. Another component (component D), emerges in the soft band only (with $S / N \sim 2.5$ ). Inspection of the image reveals that hard X-ray photons are clearly associated with the radio emission pointing eastward of the FRII core and reaching component $\mathrm{A}$, which is indeed globally harder than components $\mathrm{C}$ and $\mathrm{D}$. The $\mathrm{X}$-ray photometry of the four components is presented in Table 2. 

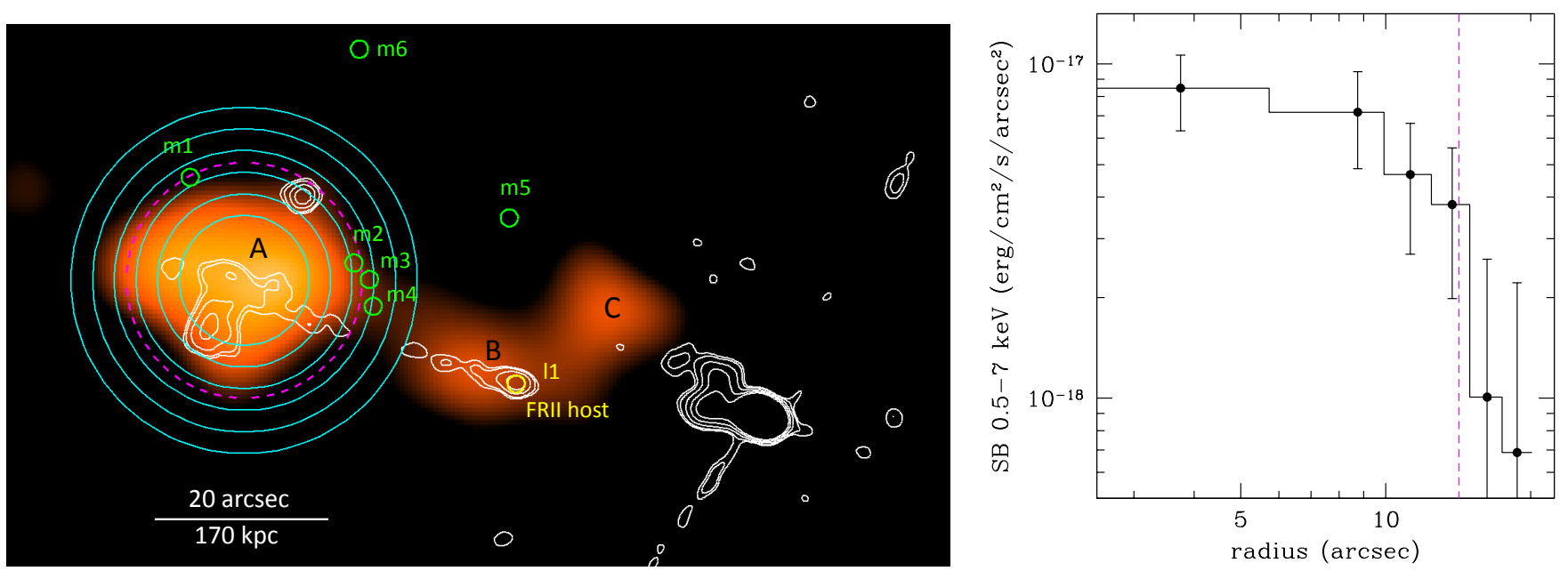

Fig. 8. Left: point-source subtracted and smoothed Chandra/ACIS-I image in the $0.5-7 \mathrm{keV}$ band. The main components A, B, and C of the diffuse $\mathrm{X}$-ray emission are marked. Galaxies at $z=1.69$ are labeled as in Fig. 1 and Table 1 ( $l 2$ falls outside this image). VLA radio contours at $1.4 \mathrm{GHz}$ are overplotted in white (same levels as in Fig. 7): they show the full morphology (with jet and lobes) of the FRII radio galaxy and an additional radio source that is also possibly part of the overdensity (see text). The annuli used to compute the surface brightness profile of component $\mathrm{A}$ (see right panel) are shown in cyan. The dashed magenta circle (14" radius around the X-ray centroid) shows the location of the overdensity galaxies $m 1-m 4$. Right: surface brightness profile of the diffuse component A as measured on the (point-source subtracted) Chandra $0.5-7 \mathrm{keV}$ image before smoothing, and using the extraction annuli shown in the left panel. The radial profile is background subtracted and a power-law spectrum with $\Gamma=1.6$ is assumed to convert count rates into fluxes. The radius at which the $m 1-m 4$ galaxies are found is shown by the magenta vertical line. The X-ray surface brightness sharply decreases at this radius, and is consistent with zero beyond it.

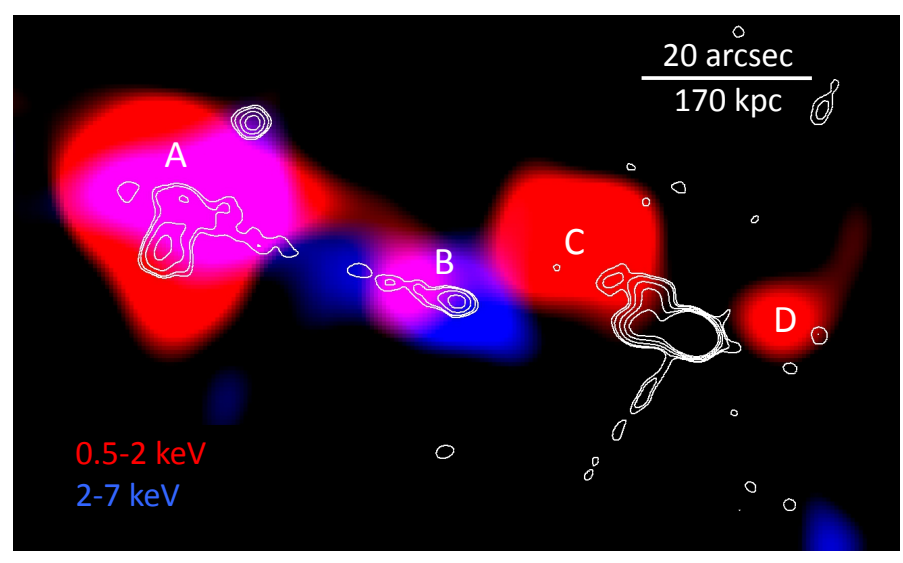

Fig. 9. Point-source subtracted and smoothed Chandra/ACIS-I X-ray color image of the diffuse emission (see text for details). Soft $(0.5-$ $2 \mathrm{keV})$ and hard (2-7 keV) X-rays are shown in red and blue, respectively. The $\mathrm{X}$-ray emission is shown down to a $\sim 2.5 \sigma$ significance level. Radio contours are shown in white.

Nanni et al. (2018) discussed the origin of component A and proposed that it can be either associated with feedback produced by the $z=6.3$ quasar (which is located in projection just above this structure) on its close environment, or with the eastern lobe and jet of the FRII radio galaxy. We extracted and analyzed the X-ray spectrum of component A $(\sim 100$ net counts in the full band), but could not identify any significant spectral feature to measure its redshift.

Based on an in-depth analysis of the structure of the diffuse X-ray emission, we here suggest that this is likely to be produced at $z=1.7$. First, as discussed above and reported in Table 2, in addition to the main component A, we detected three more significant spots of diffuse X-ray emission across the whole structure of the FRII and of the related galaxy overdensity. The presence of multiple spots of diffuse X-ray radiation that are located in the same region covered by the FRII and by the overdensity structure provides a strong indication that diffuse X-rays are actually produced at $z=1.7$. Second, when X-ray point-like sources are removed from Chandra images, and, in particular, the nuclear emission of the $z=6.3$ QSO is removed, no significant diffuse X-ray emission reaches the position of the QSO (see, e.g., Fig. 12). Despite some residual uncertainties that are related to the limited photon statistics, to the point source subtraction process, and to the smoothing process, the lack of any significant diffuse X-ray structure directly emanating from the QSO location argues against an origin at $z=6.3$. In contrast, the eastern radio lobe of the FRII appears extremely well centered on component $\mathrm{A}$, and it even bends southward after reaching the centroid of the diffuse X-ray emission: this also suggests some relation between the X-ray and radio data. Because of the above arguments we then assume here that most of the observed diffuse $\mathrm{X}$-rays are produced at $z=1.7$.

We first fit component A with a thermal model (APEC in XSPEC) with metal abundances fixed to $0.3 \times$ solar, as is typically measured in galaxy clusters (Balestra et al. 2007). The spectrum of component $\mathrm{A}$ is rather hard (see the photometry in Table 2), and only a lower bound to the gas temperature can be obtained ( $T \gtrsim 5 \mathrm{keV}$ at $2 \sigma)$. The total $\mathrm{X}$-ray luminosity is $L_{2-10} \sim 4 \times 10^{43} \mathrm{erg} \mathrm{s}^{-1}$ in the $2-10 \mathrm{keV}$ rest-frame.

Components $\mathrm{C}$ and $\mathrm{D}$ are instead softer, and in fact are not detected in the hard band. Their emission $(\sim 21$ and $\sim 12$ net counts, respectively) is well fit by an APEC model with $T \approx$ $1 \mathrm{keV}$ (see Table 2; a fit with a power law returns implausibly steep photon indices, $\Gamma \sim 4-5$ ). The $2-10 \mathrm{keV}$ rest-frame luminosity of components $\mathrm{C}$ and $\mathrm{D}$ is $L_{2-10} \sim 1.3 \times 10^{43} \mathrm{erg} \mathrm{s}^{-1}$ and $\sim 6 \times 10^{42} \mathrm{erg} \mathrm{s}^{-1}$, respectively.

The hard spectrum of component A might indicate that nonthermal processes such as synchrotron radiation or inverse Compton scattering of cosmic microwave background (CMB) photons (IC-CMB) by the relativistic electrons in the lobe provide a non-negligible contribution to its total emission (e.g., Smail et al. 2012). This hypothesis is discussed in Sect. 5.3. 
Table 2. Diffuse X-ray emission components.

\begin{tabular}{|c|c|c|c|c|c|c|c|c|c|c|c|}
\hline \multirow{2}{*}{$\begin{array}{l}\text { ID } \\
\text { (1) }\end{array}$} & \multirow{2}{*}{$\begin{array}{l}\text { RA } \\
\text { (2) }\end{array}$} & \multirow{2}{*}{$\begin{array}{l}\text { Dec } \\
(3)\end{array}$} & \multicolumn{3}{|c|}{ Net counts } & \multirow{2}{*}{$\begin{array}{c}R \\
\left({ }^{\prime \prime}\right) \\
(7) \\
\end{array}$} & \multirow{2}{*}{$\begin{array}{l}\Gamma \\
(8) \\
\end{array}$} & \multirow{2}{*}{$\begin{array}{c}k T \\
(\mathrm{keV}) \\
(9)\end{array}$} & \multirow{2}{*}{$\begin{array}{c}f_{0.5-7} \\
\left(10^{-15} \mathrm{cgs}\right) \\
(10)\end{array}$} & \multirow{2}{*}{$\begin{array}{c}L_{2-10} \\
\left(10^{43} \mathrm{erg} \mathrm{s}^{-1}\right) \\
(11)\end{array}$} & \multirow{2}{*}{$\begin{array}{c}C / \text { d.o.f. } \\
\text { (12) }\end{array}$} \\
\hline & & & $\begin{array}{c}\text { Full } \\
(4)\end{array}$ & $\begin{array}{c}\text { Soft } \\
(5)\end{array}$ & $\begin{array}{l}\text { Hard } \\
(6)\end{array}$ & & & & & & \\
\hline A & $\begin{array}{c}\text { 10:30:27.4 } \\
" ~\end{array}$ & $\begin{array}{c}+05: 24: 39.5 \\
"\end{array}$ & $\begin{array}{c}110 \pm 20 \\
"\end{array}$ & $62 \pm 12$ & $48 \pm 17$ & $\begin{array}{c}14 \\
"\end{array}$ & $\begin{array}{c}1.64_{-0.35}^{+0.39} \\
-\end{array}$ & $\begin{array}{l}- \\
>5\end{array}$ & $\begin{array}{l}3.7 \\
3.6\end{array}$ & $\begin{array}{l}4.3 \\
4.4\end{array}$ & $\begin{array}{l}210.7 / 228 \\
211.5 / 228\end{array}$ \\
\hline B & 10:30:25.5 & $+05: 24: 29.4$ & $61 \pm 12$ & $11 \pm 6$ & $50 \pm 11$ & 8 & $0.06_{-0.44}^{+0.36}$ & - & 2.9 & 1.6 & $81.5 / 120$ \\
\hline $\mathrm{C}$ & $10: 30: 24.4$ & $+05: 24: 37.1$ & $24 \pm 10$ & $21 \pm 6$ & $3 \pm 7$ & 10 & - & $0.63_{-0.17}^{+0.28}$ & 2.1 & 1.3 & $14.3 / 29$ \\
\hline $\mathrm{D}$ & $10: 30: 22.7$ & $+05: 24: 27.9$ & $12 \pm 8$ & $12 \pm 5$ & $0 \pm 6$ & 6 & - & $0.78_{-0.20}^{+0.84}$ & 0.8 & 0.6 & $20.4 / 20$ \\
\hline
\end{tabular}

Notes. Columns: (1) component identifier; (2) and (3) $0.5-7 \mathrm{keV}$ centroid coordinates; (4), (5), (6) net counts in the 0.5-7 keV, 0.5-2 keV and 2-7 keV band, respectively; (7) extraction radius adopted for the photometric and spectral analysis; (8) best-fit photon index obtained with a power-law model (see text for details); (9) best-fit temperature obtained with a thermal model (see text for details); (10) $0.5-7 \mathrm{keV}$ observed flux; (11) $2-10 \mathrm{keV}$ rest-frame luminosity; and (12) best-fit statistics over degrees of freedom. All errors are given at the $1 \sigma$ level.

As shown in Fig. 9, the soft X-ray emission seen in component $\mathrm{A}$ appears to be more extended than the hard emission, supporting the idea that we may be seeing a mixture of IC-CMB emission in its center, and softer thermal emission on larger scales. In this case, the actual values of the gas temperature and luminosity of component A may be lower than those reported in Table 2. The origin of the diffuse X-ray emission, especially in component A, is discussed in more detail in Sects. 5.3 and 5.4.

We finally inspected component $\mathrm{B}$, which coincides with the radio jet emission and features hard $\mathrm{X}$-ray emission, as is readily apparent from Fig. 9. It contains $\sim 60$ net counts in the $0.5-$ $7 \mathrm{keV}$ band, and $\sim 80 \%$ of them are at $E>2 \mathrm{keV}$. A powerlaw fit returns a flat photon index $\Gamma=0.1 \pm 0.4$ and a flux of $f_{0.5-7}=2.9 \times 10^{-15} \mathrm{erg} \mathrm{cm}^{-2} \mathrm{~s}^{-1}$, corresponding to a $2-10 \mathrm{keV}$ rest-frame luminosity of $L_{2-10} \sim 1.6 \times 10^{43} \mathrm{erg} \mathrm{s}^{-1}$.

\section{Results}

\subsection{Structure of the overdensity}

The analysis of LBT/LUCI and VLT/MUSE data allowed us to confirm eight objects in the redshift interval $z=1.687-1.699$, including the FRII host at $z=1.699$ (see Table 1). Six of these objects are star-forming galaxies discovered by MUSE. The redshift distribution of the MUSE sources in the $z=0.9-2.5$ interval is shown in Fig. 3. Following a similar approach to that described in Gilli et al. (2003), we quantified the significance of the $z \sim 1.7$ redshift structure by smoothing the redshift distribution of all galaxies in the MUSE field (with a Gaussian with $\left.\sigma_{z}=0.2\right)$ and considering this as the MUSE background redshift distribution (red curve in Fig. 3). The Poisson probability of observing $N_{\mathrm{g}}=6$ galaxies when $N_{\mathrm{bkg}}=0.26$ are expected within $\Delta z<0.01$ is lower than $3.5 \times 10^{-7}$. As cosmic variance might be an issue on small fields such as the one studied here (Moster et al. 2011), we verified whether smoothing the observed redshift distribution provides a biased estimate of the shape and normalization of the "true" background galaxy distribution, which in turn would alter the significance of our measurement. We then downloaded the spectroscopic catalog obtained from the $3^{\prime} \times 3^{\prime}$ MUSE observation of the Hubble Ultra Deep Field (HUDF; Beckwith et al. 2006) consisting of nine MUSE pointings of $10 \mathrm{~h}$ each (Bacon et al. 2017; Inami et al. 2017), and smoothed the MUSE HUDF redshift distribution to obtain a more accurate estimate of the average background shape. This curve was then renormalized to the average number of galaxies expected in a single MUSE pointing of the HUDF. We note that if anything, this procedure may somewhat overestimate the average galaxy background density because the HUDF observations are deeper than those in the J1030 field. The derived significance of the $z=1.7$ overdensity may then be regarded as conservative. The obtained distribution was found to be very similar to that shown in Fig. 3, and our estimates of the level and significance of our $z=1.7$ overdensity are therefore strengthened by adopting a more accurate background distribution.

The structure at $z \sim 1.7$ is highly significant, and corresponds to an overdensity of $\delta_{\mathrm{g}}=N_{\mathrm{g}} / N_{\mathrm{bkg}}-1=22$. This in turn indicates that the FRII radio galaxy is the signpost of a high- $z$ large-scale structure (see Sect. 5.1 for an estimate of the structure mass). Most overdensity members lie at $<0.4 \mathrm{Mpc}$ projected separations from the FRII host, i.e. where MUSE data are available. However, the serendipitous detection of another overdensity member in the LUCI slit at $0.8 \mathrm{Mpc}$ separation from the FRII host in the opposite direction suggests that the whole structure extends for at least 1.2 projected Mpc. The existence of other candidate members at $0.4-0.7 \mathrm{Mpc}$ projected separation from the FRII host is indicated by the joint X-ray spectral and photometric redshift analysis of Chandra sources in the field (Peca et al., in prep.). The actual size of the structure can then only be established by further spectroscopic observations.

In Fig. 4 we show the distribution of the eight overdensity members in (rest-frame) velocity space. The velocity offset is computed with respect to the median redshift of the sources. All objects lie within $\Delta v=1300 \mathrm{~km} \mathrm{~s}^{-1}$, and their velocity dispersion (computed with the gapper method, Beers et al. 1990) is $325 \pm 226 \mathrm{~km} \mathrm{~s}^{-1}$, which decreases to only $121 \pm 68 \mathrm{~km} \mathrm{~s}^{-1}$ if the object at lower redshift ( $m 6$ at $z=1.6871, \Delta v \sim-1060 \mathrm{~km} \mathrm{~s}^{-1}$ in Fig. 4) is neglected. When only the six MUSE sources are considered, the velocity dispersion is $355 \pm 300 \mathrm{~km} \mathrm{~s}^{-1}$, which decreases to $85 \pm 56 \mathrm{~km} \mathrm{~s}^{-1}$ when $m 6$ is removed. These values are significantly lower than what is measured for massive galaxy clusters (e.g., $\sim 1000 \mathrm{~km} \mathrm{~s}^{-1}$ for $M \sim 10^{15} M_{\odot}$, Rosati et al. 2002) and similar to what is measured in low-mass groups with $M \sim 10^{13} M_{\odot}$ (Mulchaey 2000). In Sect. 5.1 we derive a lower limit to the total mass of our system of $M \sim 1.5-2 \times 10^{13} M_{\odot}$. As discussed above, the size, and hence the mass, of the structure is likely much larger than what can be estimated based on the MUSE data alone. The measured low-velocity dispersion may then indicate that the system is still far from virialization. By assuming that peculiar velocities are negligible, we therefore converted the redshift differences among the overdensity members into radial separations, which are also reported in Fig. 4. All objects fall within a radial separation of $\sim 7$ physical Mpc. 
Also, the four objects $m 1-m 4$ at the boundary of component A of the diffuse X-ray emission lie within $\Delta z=0.0007$, that is, within 450 radial $\mathrm{kpc}$, and the three of them falling within $60 \mathrm{kpc}$ in the plane of the sky ( $m 2-m 4$, see Fig. 8 left) also lie within $\Delta z=0.0001$, that is, within $63 \mathrm{kpc}$ in the radial direction. There are obvious uncertainties in this estimate. On the one hand, if the structure is caught far from virialization and at the beginning of its collapse phase, coherent galaxy motions may lead to an underestimate of its true radial dimension. On the other hand, errors in the redshift measurement likely inflate the true radial separations. With these caveats in mind, we note that the radial separation of the galaxies falling at the edge of component $\mathrm{A}$ of the diffuse X-ray emission is comparable with the transverse dimension of the latter.

\subsection{Star formation, ages, and masses of the overdensity members}

We computed the extinction-corrected star formation rate of the MUSE overdensity members $m 1-m 6$ starting from their absolute UV magnitude at $2800 \AA$ rest-frame $M_{2800}$. To determine $M_{2800}$, we integrated the flux of their VLT/MUSE spectra in a bandwidth of $200 \AA$ centered on $2800 \AA$. The absolute flux calibration of the MUSE data is very good (Kreckel et al. 2017), and the absence of slit losses allows us to confidently measure the total UV flux emerging from the galaxies (as also demonstrated by the excellent agreement between the optical magnitudes obtained from broad-band photometry and the fluxes measured by MUSE at different wavelengths). To convert the far-UV (FUV) luminosity into the ongoing star formation rate (SFR) we used the conversion factor $K_{\mathrm{FUV}} \equiv \mathrm{SFR} / L_{v}(\mathrm{FUV})=1.3 \times 10^{-28}$ for $Z=Z_{\odot}$ (Madau \& Dickinson 2014), where the SFR is expressed in units of $M_{\odot} \mathrm{yr}^{-1}$ and $L_{v}(\mathrm{FUV})$ in units of $\operatorname{erg~s}^{-1} \mathrm{~Hz}^{-1}$. The FUV continuum slope was measured in the range 1920-2175 rest-frame, that is, $5175-5865 \AA$ observed frame, which is relatively free from intense sky lines. To transform the continuum slope parameter measured in this range into the classical UV continuum slope $\beta$ (where $f_{\lambda} \propto \lambda^{\beta}$ ), we adopted the relations proposed by Noll \& Pierini (2005). Finally, the intrinsic SFR was computed taking into account the dust-extinction correction adopting the definition $\log \left(\mathrm{SFR}_{\text {tot }}\right)=\log \left(\mathrm{SFR}_{\mathrm{UV}}\right)+0.4 \times A_{\mathrm{IRX}}$ (Nordon et al. 2013), where $\mathrm{SFR}_{\mathrm{UV}}$ is the uncorrected star formation rate and $A_{\mathrm{IRX}}$ is the effective UV attenuation derived from the far-infrared (FIR) to UV luminosity ratio. Here we used the $A_{\text {IRX }}-\beta$ relationship derived by Talia et al. (2015). All these measurements are presented in Table 3 .

The overdensity members $m 1-m 6$ have generally blue spectra $(\beta \sim-1.3$ to -2.7$)$ and are actively forming stars, with extinction-corrected SFRs in the range $\approx 8-60 M_{\odot} \mathrm{yr}^{-1}$ (see Table 3 ). For the serendipitous LUCI source $l 2$, we derived a star formation rate of $>5 M_{\odot} \mathrm{yr}^{-1}$ based on its $\mathrm{H} \alpha$ luminosity (e.g., Kennicutt \& Evans 2012). We consider the SFR measured for $l 2$ as a lower limit because we did not apply any correction for the extinction. We did not find any evidence in our LBT/LBC and CFHT/WIRCam images for objects with optical or near-IR colors that would be consistent with a sequence of red passive galaxies at $z \sim 1.6-1.7(i-J>1.5$, Chan et al. 2018), except for the optically faint $\left(i_{\mathrm{AB}} \sim 25.4\right)$ radio object shown in Fig. 12 (for which we measured a photometric redshift consistent with 1.7 , see Sect. 5.2). This again suggests that the structure is young and likely not yet virialized, as often found for protoclusters around HzRGs (Overzier et al. 2005; Kotyla et al. 2016).

We used the STARLIGHT full-spectrum fitting code (Cid Fernandes et al. 2005) to recover the star formation history of galaxies $m 1-m 6$. STARLIGHT fits an observed spectrum with a superposition of synthetic simple stellar populations (SSPs) of various ages and metallicities, producing a best-fit spectrum. In particular, it provides the light and mass contribution of each synthetic SSP to the best-fit model at a user-defined normalization wavelength (set to $2500 \AA$ in our fits). This allowed us to recover the galaxy star formation history as the distribution of the SSP contributions as a function of their age. We adopted Bruzual \& Charlot (2003) SSP models (updated to 2016 - BC16 hereafter), which extend down to $1000 \AA$ with a resolution $\Delta \lambda=1 \AA F W H M$. We downgraded the spectral resolution of the BC16 models to that of the observed spectra, which is $\lambda / R /(1+z) \sim 2.5 \AA$, considering $R \sim 1000, z=1.7$ and an average observed $\lambda \sim 6500 \AA$. We adopted a grid of metallicities that reached from $0.005 Z_{\odot}$ to $5 Z_{\odot}$ and ages from $100 \mathrm{Myr}$ to $4 \mathrm{Gyr}$, which corresponds to the age of the Universe at $z=1.7$. Because the synthetic SSPs only model the stellar contributions to the UV flux and do not account for nebular emission or absorption from the interstellar medium (ISM), we performed the spectral fitting by masking the strongest nebular emission lines (i.e., CIII] $\lambda \lambda 1906.68,1908.68$ and CII] $\lambda 2326.00$ ) and absorption lines including ISM absorption (i.e., AlIII and FeII absorption lines in the range 1850-2850 $\AA$, see Table 1 in Talia et al. 2012). Significant episodes of star formation as young as a few megayears are found in all galaxies. Furthermore, in $m 1$ and in $m 4$ these recent bursts produce most of the optical and UV light.

We derived the stellar masses $M_{*}$ of $m 1-m 6$ by means of an SED fit using Hyperzmass, a modified version of the Hyperz code (see, e.g., Bolzonella et al. 2010). The fit is based on Bruzual \& Charlot (2003) SSPs assuming different star formation histories (either an exponentially declining or a constant SFR). Reddening was also introduced following Calzetti et al. (2000). We collected all photometric data points available for $m 1-m 6$, from the $U$-band to IRAC 3.6 and $4.5 \mu \mathrm{m}$. From 6 to 12 photometric detections at different wavelengths are available for our sources. We verified that in the MUSYC catalog the photometry of a few sources is highly inaccurate because of the contamination from the bright foreground star, in particular in the $i$ and $z$ bands. When available, we then used HST photometry in the F775W and F850LP filters (together with the F160W filter $)^{3}$. The stellar masses obtained for $m 1-m 6$ are reported in Table 3. Two examples of an SED fit (for $m 3$ and $m 4$ ) are shown in Fig. 10. The best-fit SFRs obtained with the SED fitting agree within the errors with those derived from the UV spectra. The only discrepancy is found for $m 6$, for which the SED fit derived a ten times lower SFR than what was estimated from the fit to the UV spectrum, where a relatively flat continuum was interpreted as a highly extincted starburst. The SED fit instead reveals a strong Balmer break outside the wavelength range covered by the UV spectrum, suggesting that the red spectral color is instead due to evolved stellar populations. For $m 6$ we then report the SFR measured with Hyperzmass rather than from the UV spectral fit. In Table 3 we also provide the specific star formation rates $\left(\mathrm{sSFR}=\mathrm{SFR} / M_{*}\right.$ ) obtained by combining the results of the UV spectral and SED fit.

\subsection{Power, orientation, and nuclear obscuration of the FRII}

Our Chandra spectral analysis indicates that the AGN powering the FRII radio-galaxy is a heavily obscured Compton-thick

\footnotetext{
Based on the Hubble Source Catalog, see: https://archive.
} stsci.edu/hst/hsc/ 
Table 3. Properties of MUSE members of the overdensity.

\begin{tabular}{|c|c|c|c|c|c|}
\hline (1) & $\begin{array}{c}M_{2800} \\
(\mathrm{AB}) \\
(2)\end{array}$ & $\begin{array}{l}\beta \\
(3) \\
\end{array}$ & $\begin{array}{c}\mathrm{SFR} \\
\left(M_{\odot} \mathrm{yr}^{-1}\right) \\
(4)\end{array}$ & $\begin{array}{c}\log \left(M_{*}\right) \\
\left(M_{\odot}\right) \\
(5)\end{array}$ & $\begin{array}{c}\log (\mathrm{sSFR}) \\
\left(\mathrm{Gyr}^{-1}\right) \\
(6)\end{array}$ \\
\hline$m 1$ & -19.52 & -1.33 & $20.2 \pm 3.4$ & & $0.94 \pm 0.55$ \\
\hline$m 2$ & -19.53 & -1.45 & $18.1 \pm 3.4$ & $9.69_{-0.04}^{+0.09}$ & $0.57 \pm 0.11$ \\
\hline$m 3$ & -21.25 & -1.88 & $57.1 \pm 6.2$ & $10.09_{-0.07}^{+0.05}$ & $0.67 \pm 0.08$ \\
\hline$m 4$ & -20.08 & -2.22 & $13.8 \pm 1.1$ & $9.33_{-0}^{+0}$ & $0.81 \pm 0.23$ \\
\hline$m 5$ & -19.96 & -2.70 & $7.6 \pm 1.1$ & $9.54_{-0.36}^{+0.16}$ & $0.34 \pm 0.28$ \\
\hline$m 6$ & -19.94 & -0.35 & $13.1 \pm 1.0^{(a)}$ & $10.48_{-0.01}^{+0.04}$ & $-0.37 \pm 0.04$ \\
\hline
\end{tabular}

Notes. Columns: (1) source identifier; (2) absolute magnitude at $2800 \AA$ rest-frame (not corrected for extinction); (3) slope of the optical spectrum $\left(f_{\lambda} \propto \lambda^{\beta}\right)$; (4) extinction-corrected star formation rate based on the UV spectrum; (5) stellar mass; and (6) specific star formation rate. ${ }^{(a)}$ Based on the SED fitting because it provides a more reliable estimate for this source (see text for details).

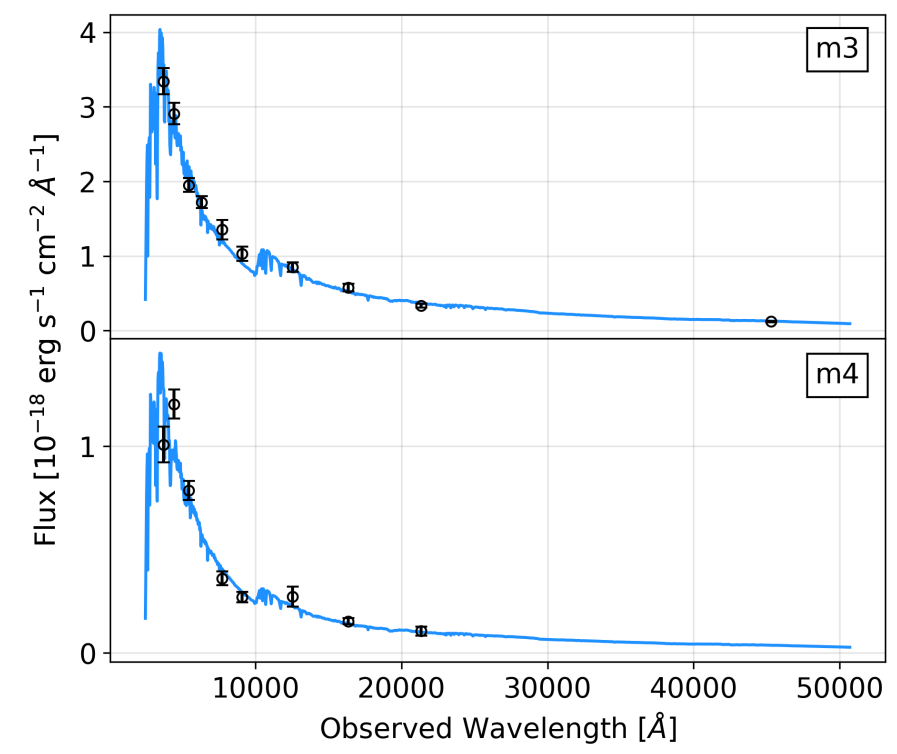

Fig. 10. Spectral energy distribution of the overdensity galaxies $m 3$ and $m 4$ and best-fit model.

QSO (XID189). The obscuration measured in the X-rays is consistent with the type 2 optical classification derived from the LUCI spectrum. According to the classic unification schemes, the highest column densities are expected in systems where the inclination angle $\theta$ between the axis of an approximately toroidal parsec-scale distribution of obscuring gas and our line of sight is $\sim 90 \mathrm{deg}$, that is, when the system is seen edge-on. In the hypothesis that the obscuring torus and radio jet are coaxial, we can estimate $\theta$ based on the radio data. We used the VLA radio images to estimate the flux ratio $R_{\text {jet }}$ between the jet vs. counter-jet emission at the smallest possible scales and obtained a joint constraint on the jet velocity $\beta=v / c$ and inclination angle $\theta$ (assuming equal power jets) of

$R_{\text {jet }}=[(1+k) /(1-k)]^{p+\alpha_{\text {jet }}}$,

where $k \equiv \beta \cos (\theta), \alpha_{\text {jet }}$ is the spectral index of the jet $\left(f_{v} \propto\right.$ $\left.v^{-\alpha_{\mathrm{jet}}}\right)$ and $p$ is the Doppler boost exponent. We measured $R_{\text {jet }}$ in the range 1.42-1.65, which for typical values of $\alpha_{\text {jet }} \sim 0.5$ and assuming a continuous jet with $p=2$ translates into $k \sim$ $0.07-0.1$.

Another constraint on $k$ can be obtained by comparing the observed Doppler-boosted power in the core $P_{\text {core }}^{\text {obs }}$ with an estimate of the intrinsic (not boosted) core power $P_{\text {core }}^{\text {int }}$ (see, e.g., Cohen et al. 2007):

$R_{\text {core }} \equiv P_{\text {core }}^{\text {obs }} / P_{\text {core }}^{\text {int }}=\delta^{p+\alpha_{\text {core }}}$,

where $\delta=\sqrt{\left(1-\beta^{2}\right)} /(1-k)$ is the relativistic Doppler factor. By combining Eqs. (1) and (2), we can then derive both $\beta$ and $k$, and in turn, the inclination angle $\theta=\operatorname{arcos}(k / \beta)$.

An estimate of the intrinsic core power $P_{\text {core }}^{\text {int }}$ can be obtained from the total extended radio power by means of the $P_{\text {tot }}(408 \mathrm{MHz})-P_{\text {core }}^{\text {int }}(5 \mathrm{GHz})$ correlation described, for instance, by Giovannini et al. (2001). Based on the $150 \mathrm{MHz}$ data of the GMRT TGSS survey ${ }^{4}$, we measured a total flux in the two summed radio lobes equal to $\sim 160 \mathrm{mJy}$ (within the $3.5 \sigma$ contours). For a radio spectral index of $\alpha_{\text {tot }}=0.8\left(f_{v} \propto v^{-\alpha_{\text {tot }}}\right)$ that we derived from the comparison between the TGSS data and the NVSS data at $1.4 \mathrm{GHz}$ (see Nanni et al. 2018 for details), the TGSS flux converts into a total rest-frame extended luminosity at $408 \mathrm{MHz}$ of $P_{\text {tot }}(408 \mathrm{MHz}) \sim 10^{26} \mathrm{~W} \mathrm{~Hz}^{-1} \mathrm{sr}^{-1}$. This value, when combined with the $\mathrm{H} \alpha$ luminosity measured in Sect. 2, nicely places XID189 on the total radio power vs. H $\alpha$ luminosity correlation reported by Zirbel \& Baum (1995) for powerful FRII radio galaxies.

The observed core power $P_{\text {core }}^{\text {obs }}$ at $5 \mathrm{GHz}$ was assumed to be equal to that observed at $1.4 \mathrm{GHz}$ (i.e., we assumed a typical spectral slope of $\alpha_{\text {core }}=0.0$ because this cannot be derived directly from our data), which gives $R_{\text {core }} \equiv P_{\text {core }}^{\text {obs }} / P_{\text {core }}^{\text {int }} \sim$ $0.9-1.2$. Based on Eqs. (1) and (2), we finally derived $\beta \sim$ $0.4-0.5$ and $\theta \sim 70-80 \mathrm{deg}$. This indicates that the system is truly seen almost edge-on, as expected for this heavily obscured FRII nucleus.

The absorption-corrected luminosity in the rest-frame $2-10 \mathrm{keV}$ band is $L_{2-10}=1.3 \times 10^{44} \mathrm{erg} \mathrm{s}^{-1}$, which, adopting a bolometric correction of 30, as appropriate for these X-ray luminosities (e.g., Marconi et al. 2004), translates into a total radiated luminosity of $L_{\mathrm{rad}} \sim 4 \times 10^{45} \mathrm{erg} \mathrm{s}^{-1}$. This is well into the QSO regime. To compute the bulk kinetic power of the FRII jet, we assumed it to be proportional to the total radio luminosity of the lobes, following Willott et al. (1999) (see also Hardcastle et al. 2007; Shankar et al. 2008):

$P_{\text {jet }}=3 \times 10^{45} f^{3 / 2} L_{151}^{6 / 7} \mathrm{erg} \mathrm{s}^{-1}$,

where $L_{151}$ is the total observed luminosity at $151 \mathrm{MHz}$ in units of $10^{28} \mathrm{~W} \mathrm{~Hz}^{-1} \mathrm{sr}^{-1}$ and the factor $f$ encapsulates all the systematic uncertainties on the system geometry and environment and on the jet composition (see Willott et al. 1999, for details). Based on the TGSS data and on the spectral slope mentioned above, we derived a rest-frame extended radio luminosity of $L_{151} \sim 2.1 \times 10^{26} \mathrm{~W} \mathrm{~Hz}^{-1} \mathrm{sr}^{-1}$. By further assuming $f=15$, which is a reasonable value for FRII radio galaxies (see Hardcastle et al. 2007), from Eq. (3) we derived a total jet kinetic power of $P_{\text {jet }} \sim 6.3 \times 10^{45} \mathrm{erg} \mathrm{s}^{-1}$. Our measurements indicate that $P_{\text {jet }} \sim 1.5 \times L_{\text {rad }}$, that is, the energy released in the form of baryons in the jet is equal to or even higher than that released in the form of photons by the accretion disk, as is generally found for AGN with powerful radio jets (Ghisellini et al. 2014). The effects of this energy release on the surrounding environment is discussed in Sect. 5.4.

\footnotetext{
4 http://tgssadr.strw.leidenuniv.nl/doku.php
} 


\section{Discussion}

\subsection{Total mass of the structure}

Measuring the total mass of a system that is likely far from virialization is not an easy task. A rough estimate of the dark matter mass contained in the structure can be obtained by means of the measured galaxy overdensity: $M_{\mathrm{tot}}=\bar{\rho}_{\mathrm{m}} V\left(1+\delta_{\mathrm{m}}\right)$, where $\bar{\rho}_{\mathrm{m}}$ is the average density of the Universe at the redshift of the structure, $V$ is its volume, and $\delta_{\mathrm{m}}$ is the dark matter overdensity. In order to have some control on the volume spanned by our observations, we considered in our estimate only the six galaxies found by MUSE. The volume $V$ is assumed to be a box of dimensions $0.5 \times 0.5 \times 6.3=1.6$ proper $\mathrm{Mpc}^{3}$, that is, the MUSE FoV $\left(0.5 \times 0.5 \mathrm{Mpc}^{2}\right.$ at $\left.z=1.7\right)$ multiplied by the maximum radial separation of the MUSE overdensity members (i.e., 6.3 Mpc assuming that peculiar velocities are negligible; see, e.g., Fig. 4). The dark matter overdensity can be derived as $\delta_{m}=\delta_{\mathrm{g}} / b$, where $\delta_{\mathrm{g}}$ is the measured galaxy overdensity $\left(\delta_{\mathrm{g}}=22\right.$, see Sect. 4.1), and $b$ is the bias of the considered galaxy population. Here we assumed $b=2$, which is appropriate for galaxies with star formation rates and redshifts similar to those of our MUSE sample (e.g., Lin et al. 2012). Under these assumptions the derived structure mass is $M=1.3 \times 10^{13} M_{\odot}$. If the system is far from virialization and still collapsing, galaxy infall motions would cause the system volume $V_{\text {app }}$ to appear smaller and the overdensity in turn higher than their true values. We tried to correct for these effects and estimated the volume compression factor $C$ following Cucciati et al. (2014). The true volume of the system is defined as $V_{\text {true }}=V_{\text {app }} / C$ (with $C<1$ ) and the true mass overdensity $\delta_{\mathrm{m}}$ is given by $1+b \delta_{\mathrm{m}}=C\left(1+\delta_{\mathrm{g}, \mathrm{obs}}\right)$, where $\delta_{\mathrm{g}, \text { obs }}$ is the observed galaxy overdensity, and $b=\delta_{\mathrm{g}} / \delta_{\mathrm{m}}$ is the true bias parameter. In the hypothesis that the system is undergoing a simple spherical collapse, the compression factor $C$ can be written as $C=1+f-f\left(1+\delta_{\mathrm{m}}\right)^{1 / 3}$ (Steidel et al. 1998), where $f(z) \approx \Omega_{\mathrm{m}}(z)^{0.6}$ for a $\Lambda$ CDM model (Lahav et al. 1991). By assuming again $b=2$ and by solving numerically, we obtained $C=0.4$, that is, $V_{\text {true }}=3.9 \mathrm{Mpc}^{3}$, and $\delta_{\mathrm{m}}=3.7$. Using these revised values, we obtained for the system a mass of $M=1.5 \times 10^{13} M_{\odot}$, very similar to what was estimated by the observed values, as the larger volume is largely compensated for by the lower overdensity. By considering that the structure likely extends far beyond the MUSE FoV (see, e.g., Fig. 1), these measurements are presumably lower limits. For comparison, the total virial mass that would be obtained for this system from the observed line-of-sight velocity dispersion of $325 \mathrm{~km} \mathrm{~s}^{-1}$ (following, e.g., Lemaux et al. 2012 and Cucciati et al. 2018) would be $\approx 2.5 \times 10^{13} M_{\odot}$. Future spectroscopic observations on wider areas are needed to properly sample the size and mass of the structure and verify whether this is the progenitor of a massive galaxy cluster $\left(M>10^{14} M_{\odot}\right)$ caught in its major assembly phase.

\subsection{Another radio galaxy in the structure?}

The VLA data showed the existence of another radio source within the region covered by the overdensity. As shown in Figs. 8 and 9, this additional radio source is compact and falls at the northern edge of component A of the diffuse X-ray emission, in between MUSE galaxies $m 1$ and $m 2$. It is therefore interesting to investigate whether it is part of the structure. The source is faint in the optical images (e.g., $r=26.18 \pm 0.13$, $i=25.35 \pm 0.13$ in the LBT/LBC catalog of Morselli et al. 2014) and has very red colors (e.g., $i-J=2.5$, using LBT/LBC and CFHT/WIRCam photometry). We computed the photometric

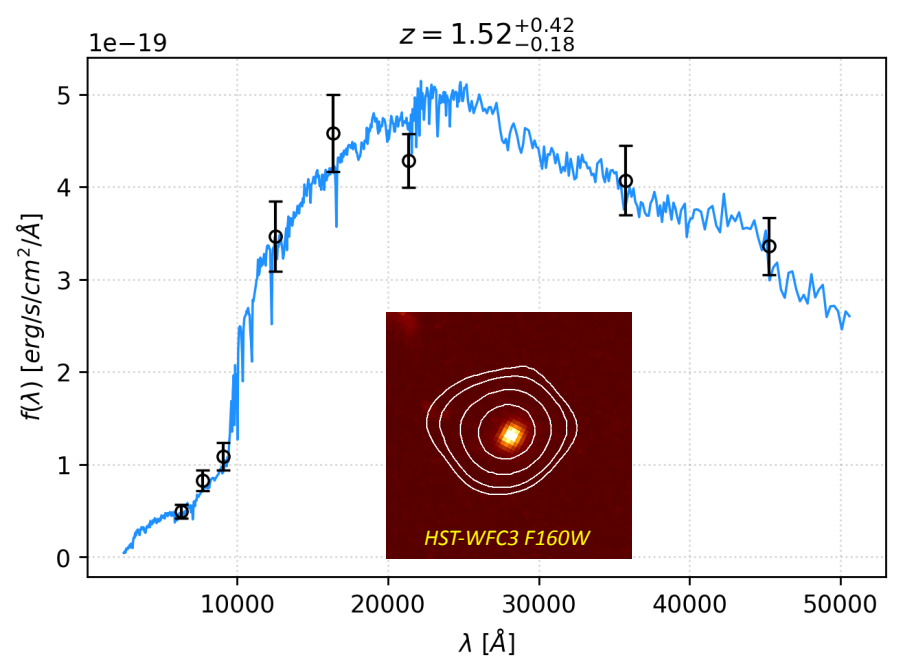

Fig. 11. Broadband SED and photometric redshift solution for the radio source at the edge of component A of the diffuse X-ray emission. The inset shows an $8^{\prime \prime} \times 8^{\prime \prime}$ cutout of the HST/WFC3 F160W image with radio contours overlaid in white (with the same levels used in Fig. 7).

redshift of the radio source by running the Hyperz code (Bolzonella et al. 2000) on the broadband photometry obtained from the LBT/LBC, WIRCam, and MUSYC catalogs. We also added IRAC photometry at 3.6 and $4.5 \mu \mathrm{m}$ as retrieved from the Spitzer public catalog available at IRSA ${ }^{5}$. The broadband photometry and associated best-fit redshift solution are shown in Fig. 11. The measured photometric redshift $z_{\text {phot }}=1.52_{-0.18}^{+0.42}$ ( $1 \sigma$ errors) is fully consistent with $z=1.69$, and the radio source is therefore an additional candidate AGN in the structure that is hosted by a passive evolved galaxy. Assuming a redshift of $z=1.69$, the total flux density of $300 \pm 30 \mu \mathrm{Jy}$ measured in the VLA images at $1.4 \mathrm{GHz}$ converts into a total radio power of $L_{1.4 \mathrm{GHz}}=4.7 \pm 0.5 \times 10^{23} \mathrm{~W} \mathrm{~Hz}^{-1} \mathrm{sr}^{-1}$, placing this source just above the knee of the radio luminosity function of radio-emitting AGN at comparable redshifts (Smolčić et al. 2017). Moreover, based on the SED fit with Hyperzmass and using $z=1.69$, we measured a stellar mass for this object of $M_{*} \sim 10^{11} M_{\odot}$. This value nicely agrees with what we derived using the tight correlation between stellar mass and observed $K$-band magnitude found by Nantais et al. (2013) for the members of two galaxy clusters at $z=0.8$ and $z=1.2$ ( $\mathrm{rms}$ of $0.14-0.19 \mathrm{dex}$ ), and rescaling to $z=1.7$. For comparison, the stellar mass that we obtained for the FRII host, that is, for the candidate brightest cluster galaxy (BCG) progenitor using its $K$-band magnitude, is only three times higher. Because of its vicinity to the bright central star, no reliable photometry can be obtained in bluer bands for the FRII host, which prevents any accurate SED fitting. If future optical and near-infrared (NIR) spectroscopy confirms that the radio source is at $z_{\mathrm{spec}}=1.7$, then it will be one of the most massive galaxies of the overdensity.

\subsection{IC-CMB as the origin of the diffuse $X$-rays?}

We here investigate the possibility that the diffuse X-ray emission seen in component $\mathrm{A}$ arises from inverse Compton scattering of $\mathrm{CMB}$ photons by the relativistic electrons of the eastern radio lobe (IC-CMB), as the energy density of the CMB steeply increases with redshift as $(1+z)^{4}$. The hard spectrum of

5 https://irsa.ipac.caltech.edu/ 


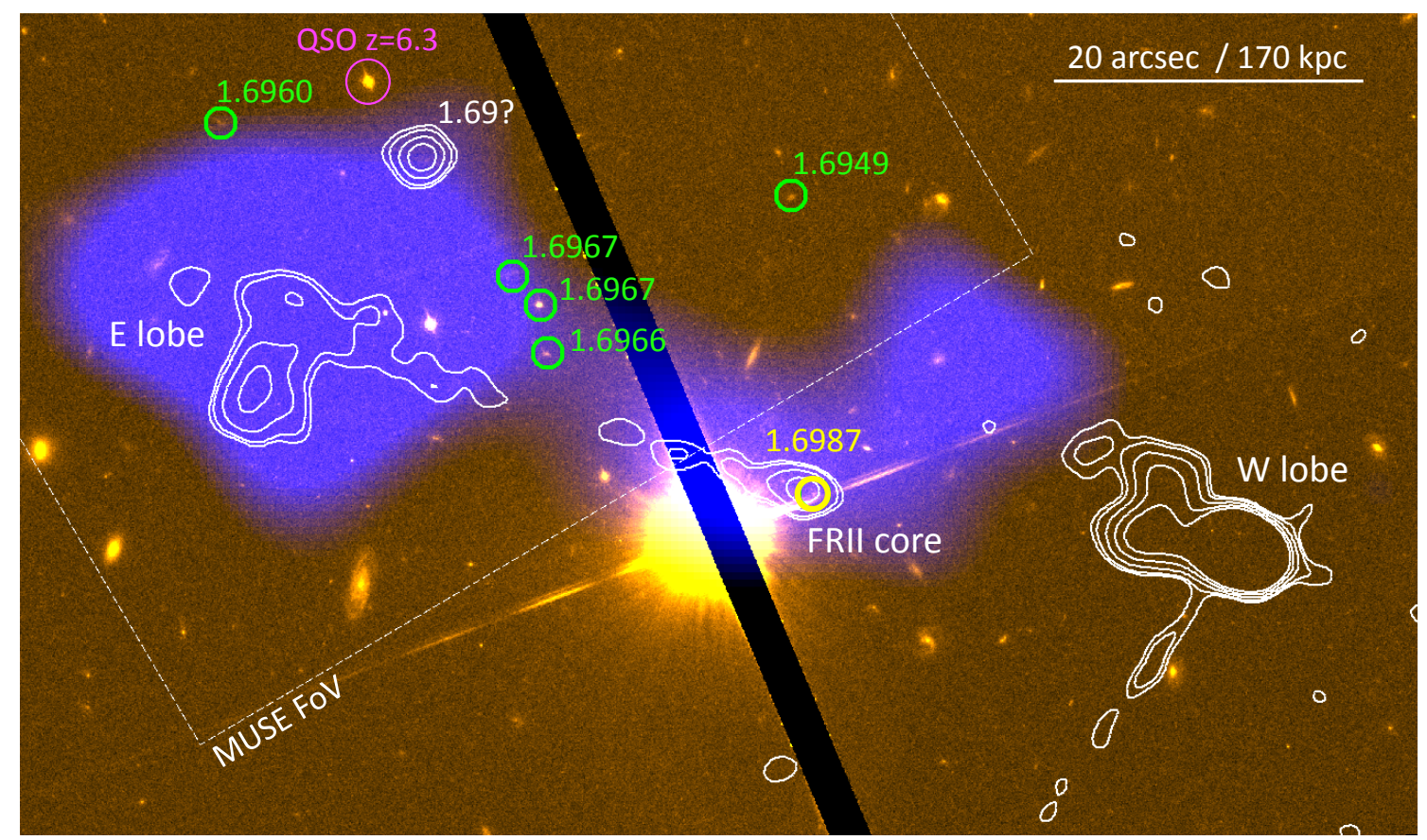

Fig. 12. HST/ACS F850LP image of the overdensity overlaid with radio contours from the VLA (in white, same levels as in Fig. 7) and the Chandra/ACIS-I smoothed image of diffuse X-ray emission (blue-violet; $0.5-7 \mathrm{keV}$ band, point sources removed). North is up and east is to the left. The dark strip running across the bright star is the gap between the two ACS CCDs. The main radio morphological features of the FRII galaxy are labeled in white. The position of the MUSE pointing is shown. The position of the FRII host and its redshift are shown in yellow. Green circles mark MUSE galaxies in the overdensity; their redshifts are as labeled. The additional radio source that may be part of the overdensity $\left(1.4<z_{\text {phot }}<1.9\right.$ at $68 \%$ confidence level) is labeled in white. The position of the $z=6.3$ QSO SDSS J1030+0524 is also marked in magenta.

component A (see Table 2) might indeed indicate a nonnegligible contribution from IC-CMB. As discussed in Nanni et al. (2018), the absence of X-rays from the western lobe, which is a factor of $>6$ times brighter in the radio, poses a challenge to this interpretation. We investigate this question in more detail below.

If the energy in the radio lobes is equally distributed between relativistic particles and magnetic field, it is possible to provide an estimate of the magnetic field at the equipartition $B_{\text {eq }}$, and in turn, of the flux density at $1 \mathrm{keV}$ expected from IC-CMB using, for instance, Eq. (11) of Harris \& Grindlay (1979):

$f_{1 \mathrm{keV}}=\frac{\left(5.05 \times 10^{4}\right)^{\alpha} C(\alpha) G(\alpha)(1+z)^{\alpha+3} f_{\mathrm{r}} v_{\mathrm{r}}}{10^{47} B_{\mathrm{eq}}^{\alpha+1}}$,

where $C(\alpha)$ and $G(\alpha)$ are tabulated constants (see, e.g., Harris $\&$ Grindlay 1979; Pacholczyk 1970) and $\alpha$ is the radio spectral index $\left(f_{\mathrm{r}} \propto v_{\mathrm{r}}^{-\alpha}\right)$. The flux densities, measured in the same region, are in cgs units.

$B_{\text {eq }}$ can be calculated using Eq. (3) of Miley (1980) and following standard prescriptions, that is, a ratio of energy in the heavy particles to that in the electrons $k=1$, a volume filling factor $\eta=1$, and an angle between the uniform magnetic field and the line of sight $\varphi=90^{\circ}$. For the eastern lobe we considered a radio spectral index of $\alpha=0.8(0.01 \mathrm{GHz}<v<100 \mathrm{GHz})$ and a flux density of $f_{\mathrm{r}}^{\text {obs }}=1.7 \mathrm{mJy}$ at $\nu_{\mathrm{r}}^{\mathrm{obs}}=1.4 \mathrm{GHz}$ as derived from the VLA maps. The emitting region ( $E$ lobe in Fig. 12) was approximated by an ellipse of angular diameters $\theta_{x} \sim 10 \operatorname{arcsec}$, $\theta_{y} \sim 12$ arcsec. The path length through the source in the radial direction was assumed equal to the angular diameter in the $x$-direction, that is, $\sim 90 \mathrm{kpc}$. We estimated a magnetic field of $B_{\text {eq }} \sim 5 \mu \mathrm{G}$, in reasonable agreement with values reported in the literature (Isobe et al. 2011). Under this condition, the ICCMB flux in the $0.5-7 \mathrm{keV}$ band (again assuming a power law of spectral index $\alpha=0.8$, i.e., a photon index of $\Gamma=1.8$, consistent within the errors with that reported in Table 2 for component A) is 60 times lower than what is observed in component A. Even considering a population of relativistic electrons missed by current VLA data and distributed over the entire region $\mathrm{A}$, the expected $\mathrm{X}$-ray flux would still be more than one dex lower than what is observed. It is nonetheless possible that the extended radio structures are not in an equipartition state, as observed in some galaxies (Migliori et al. 2007; Isobe et al. 2011). In this case, a reduction of the magnetic field by a factor of $\sim 3$ would be sufficient to obtain the observed X-ray flux in the A region. If such a deviation from equipartition is present in both radio lobes, then an X-ray flux of $\sim 10^{-15} \mathrm{erg} \mathrm{cm}^{-2} \mathrm{~s}^{-1}$ has to be expected in the western lobe, which is instead excluded by our deep Chandra data (at the $\sim 2 \sigma$ level). Therefore, to simultaneously explain the diffuse X-ray emission around the eastern lobe and its absence around the western lobe, we have to assume that (1) the magnetic field in the eastern lobe is a factor of 3 below equipartition, (2), the western lobe is instead around equipartition, and (3) low surface brightness emission that fills the entire component $\mathrm{A}$ has been missed by current radio data. We consider such a combination of requirements less probable than the shock-heating scenario discussed in the next section, but future observations in the radio band, for example, with the LOw Frequency ARray (LOFAR), will reveal whether IC-CMB is a plausible scenario as well.

\subsection{Star formation promoted by AGN feedback}

Intriguingly, the overdensity members $m 1-m 4$ (as well as the radio object at $z_{\text {phot }} \approx 1.7$ ) appear to lie at the northern boundary of component $\mathrm{A}$ of the diffuse X-ray emission, that is, the one around the eastern radio lobe of the FRII (see Figs. 8 left 
and 12). We tested whether the location of the boundary of component $\mathrm{A}$, and hence its spatial coincidence with the position of the MUSE sources in the overdensity, is a spurious effect introduced by our smoothing procedure or if it is a real feature. We computed the radial profile of the X-ray surface brightness of component $\mathrm{A}$ on the unsmoothed $0.5-7 \mathrm{keV}$ image using the series of annuli shown in Fig. 8 (left). We evaluated the background in a nearby region free from point-like or diffuse X-ray sources. The surface brightness profile, shown in Fig. 8 (right), rapidly falls off beyond $\sim 14$ arcsec from the center of component $\mathrm{A}$, and it is consistent with zero beyond that distance. Similar results are obtained for the $0.5-2 \mathrm{keV}$ image. The four overdensity members $m 1-m 4$ are all at this roll-off distance (see Figs. 8 and 12). We therefore conclude that the spatial coincidence between the boundary of the diffuse X-ray emission and the location of the four galaxies is not an artifact introduced by our smoothing procedure. Statistically, the binomial probability of finding four (or more) out of the six $z=1.69$ MUSE galaxies along that boundary (assuming it can be approximated with an annulus of radius $14^{\prime \prime}$ and width $5^{\prime \prime}$, hence representing $\sim 5 \%$ of the MUSE FoV) is very low, $P \sim 1.2 \times 10^{-4}$.

The spatial overlap between the radio lobe and the diffuse X-ray emission of component A and the location of the $m 1-m 4$ members of the overdensity, right at the edge of this diffuse $\mathrm{X}$-ray emission, led us to speculate that there is a physical connection between all these observables. For example, the eastern lobe of the radio galaxy bends toward the south, indicating that some interaction with hot gas within component $\mathrm{A}$ is plausible.

We first investigated whether, assuming that the observed $\mathrm{X}$-ray emission is mostly thermal, the jet is powerful enough to deposit the observed amount of energy into component A. Based on its measured projected size of $240 \mathrm{kpc}$, we approximated component $\mathrm{A}$ as a sphere of $120 \mathrm{kpc}$ radius. By assuming a gas temperature and density of $T=5 \mathrm{keV}$ and $n \sim 4 \times 10^{-3} \mathrm{~cm}^{-3}$, respectively (consistently with the spectral fit), we find that a total thermal energy of $E_{\mathrm{th}} \sim n V k T \sim 7 \times 10^{60} \mathrm{erg}$ is stored in component A. For comparison, the absence of X-ray emission in the western lobe $\left(f_{0.5-7}<1.4 \times 10^{-15} \mathrm{erg} \mathrm{cm}^{-2} \mathrm{~s}^{-1}\right.$ at $2 \sigma$ ) translates into a $2 \sigma$ upper limit on the gas density of $n<$ $2.5 \times 10^{-3} \mathrm{~cm}^{-3}$ (assuming that this gas has the same extension and temperature as the gas measured in the eastern lobe, and hence that the difference in the X-ray emissivity only depends on $n^{2}$ ). This limit would be even less stringent if the putative hot gas associated with the $\mathrm{W}$ lobe is not as extended as component A. We therefore conclude that the observed asymmetry in the Xray morphology may be caused by relatively small variations in the particle density of the shock-heated gas.

Both recent hydrodynamic simulations of AGN jet propagation within ICM (Bourne et al. 2019) and classic computations of energy-driven outflows produced by steady winds in astrophysical sources (Weaver et al. 1977) suggest that about $40-50 \%$ of the jet or wind power goes into gas heating, whereas the other half goes into $p d V$ work on the ambient medium. By assuming a constant jet power equal to that measured in Sect. 4.3, $P_{\text {jet }}=6.3 \times 10^{45} \mathrm{erg} \mathrm{s}^{-1}$, and that only half of it is available for gas heating, it would take $E_{\text {th }} /\left(P_{\text {jet }} / 2\right)=70 \mathrm{Myr}$ to heat the gas up to the level observed in component $\mathrm{A}$. This lifetime is perfectly consistent with the spectral ages of $\sim 70-80$ Myr recently derived from LOFAR observations at low radio frequencies of local FRII radio galaxies of similar power to XID189 (Harwood et al. 2017). We recall that because of the uncertainties in the actual gas temperature, the total thermal energy of component $\mathrm{A}$ is also uncertain. On the one hand, if $T>5 \mathrm{keV}$ (as derived from the X-ray spectral fit when a pure thermal model is used), the total thermal energy would be higher. On the other hand, because of the likely contribution of IC-CMB emission to the observed X-ray luminosity, the temperature and thermal energy of the gas in component A may have been overestimated, and the constraints on the duration of the jet activity can in turn be relaxed. These arguments suggest that the thermal energy reservoir of component A may plausibly be produced by AGN feedback.

Furthermore, if the X-ray emission of component $\mathrm{A}$ is due to a bubble of gas that is shock-heated by the FRII jet, we may wonder whether this bubble is still expanding or whether it has stalled at the observed boundary. We compared the internal gas pressure of component A $\left(P_{\text {hot }}\right)$ with that of a putative cold ambient medium within the overdensity $\left(P_{\text {cold }}\right)$, assuming this shares the same physical properties of the CGM observed in the Spiderweb protocluster, where a reservoir of $10^{11} M_{\odot}$ of cold molecular gas has been found to extend on scales of $\sim 50-70 \mathrm{kpc}$ diameter through ATCA and ALMA observations of low- $J$ CO transitions (Emonts et al. 2018). Based on these measurements, the particle density of the ambient medium would be $n \sim 7 \times 10^{-2} \mathrm{~cm}^{-3}$, and its temperature is expected to be a few tens of kelvin, which would imply $P_{\text {hot }} / P_{\text {cold }}>10^{5}$, that is, the hot gas bubble is still expanding. A similar result $\left(P_{\text {hot }} / P_{\text {cold }} \gtrsim 10^{4}\right)$ is obtained if instead the ambient medium is mostly in the form of atomic gas with temperatures $T<10^{5} \mathrm{~K}$ and column densities $N_{\mathrm{H}} \sim 10^{19-20} \mathrm{~cm}^{-2}$, similar to those found in protoclusters at $z \sim 2$ through the study of absorption lines (e.g., Ly $\alpha$, SiII, and CIV) in the rest-UV spectra of background objects (Cucciati et al. 2014; Hennawi et al. 2015). Again, with the caveat that we might have somewhat overestimated the pressure of the hot component, the bubble of hot gas is likely expanding with time.

We remark that to our knowledge, this is the first time that such a hot gas bubble inflated by AGN feedback is observed in a high- $z$ structure that is likely caught in its main assembly phase. Such a hot bubble may contribute significantly to the pre-heating of the ICM by mixing with the cooler ambient gas (see, e.g., Hillel \& Soker 2016). The rapid expansion of this bubble $(v>$ $1000 \mathrm{~km} \mathrm{~s}^{-1}$, see below) and the consequent ICM preheating on scales of up to several hundred kiloparsecs may even be favored in the early evolutionary stages of cluster assembly, when the pressure and density of the ambient gas are far lower than those of evolved and collapsed structures. All these possibilities, as well as the observational prospects of detecting these early hot gas bubbles, for instance, through the Sunyaev-Zeldovich effect (see, e.g., Ehlert et al. 2019), will be discussed elsewhere.

It is now interesting to verify whether the dimensions of the hot gas bubble are consistent with its expansion velocity and age, that is, with the AGN lifetime of $70 \mathrm{Myr}$ derived above. At $v=0.5 c$, it would only take $2 \mathrm{Myr}$ for the jet particles to move from the FRII core to the eastern lobe, therefore the bubble age can be considered equal to the AGN lifetime. Several works address the problem of expanding gas bubbles inflated by shocks produced by relativistic collimated jets or relativistic wide-angle winds launched by an AGN, using both analytic and numerical methods (Weaver et al. 1977; Costa et al. 2014; Bourne et al. 2019). We used here the formalism presented in Gilli et al. (2017), where we derived the expansion laws for bubbles that are inflated by relativistic winds launched by exponentially growing supermassive black holes. For simplicity, we assumed that a shock is generated at the center of component $\mathrm{A}$ as the relativistic jet collides with a clump of cold gas in the structure, and that this shock expands isotropically. As the cooling time of the shock-heated gas is expected to be longer than the AGN lifetime, we refer to the case of an energy-driven outflow, that is, 
to Eqs. (16) and (15) in Gilli et al. (2017), which describe the temporal evolution of the bubble radius $R(t)$ and its normalization $R_{0}$, respectively ${ }^{6}$. These equations, which were derived for radio-quiet AGN, can be extended to jetted sources by considering that the gravitational energy of the matter falling toward the black hole is dissipated not only through radiation from the accretion disk $\left(L_{\mathrm{rad}}\right)$, but also through jet launching $\left(P_{\text {jet }}\right)$. The global accretion efficiency $\epsilon_{\mathrm{tot}}$ can therefore be written as $\epsilon_{\mathrm{tot}}=\epsilon_{\mathrm{r}}+\epsilon_{\mathrm{k}}$, where $\epsilon_{\mathrm{r}}$ and $\epsilon_{\mathrm{k}}$ are the disk radiative and the jet kinetic efficiency, respectively (see, e.g., Jolley et al. 2009 and Ghisellini et al. 2013). Accordingly, the black hole mass grows from its initial value $M_{0}$ as $M(t)=M_{0} \mathrm{e}^{t / t_{\text {Sal }}}$, where the Salpeter time $t_{\text {Sal }}$ now reads: $t_{\text {Sal }}=0.45 \operatorname{Gyr}\left(\frac{\epsilon_{\mathrm{r}}}{1-\epsilon_{\mathrm{tot}}}\right) \lambda^{-1}$, where $\lambda$ is the Eddington ratio. The normalization of the bubble radius $R_{0}$ depends on several parameters: the Salpeter time $t_{\mathrm{Sal}}$, the ratio $f_{\mathrm{w}} \equiv P_{\text {kin }} / L_{\mathrm{rad}}$, the Eddington ratio $\lambda$, the initial black hole mass $M_{0}$, and the ambient gas density $\rho_{\text {gas }}$. We assumed here that the black hole is accreting at its Eddington limit $(\lambda=1)$, and that it is rapidly spinning (with, e.g., $\left.\epsilon_{\text {tot }}=0.3\right)$, as is generally thought for black holes powering jetted AGN (Blandford \& Znajek 1977; Ghisellini et al. 2014). The $L_{\text {rad }}$ and $P_{\text {kin }}$ values measured in the previous sections give $f_{\mathrm{w}} \sim 2$ (i.e., $\epsilon_{\mathrm{r}} \sim 0.1$ and $\epsilon_{\mathrm{k}} \sim 0.2$ ). For $L_{\mathrm{rad}}=4 \times 10^{45} \mathrm{erg} \mathrm{s}^{-1}$ as measured in Sect. 4.3 , we obtain $3.2 \times 10^{7} M_{\odot}$ for the mass of the black hole powering the FRII galaxy, which for an accretion time of $70 \mathrm{Myr}$ must then have grown from an initial mass $M_{0}=6 \times 10^{6} M_{\odot}$. Finally, we assumed an ambient gas with uniform density of $\rho_{\text {gas }} \sim 2 \times 10^{-28} \mathrm{~g} \mathrm{~cm}^{-3}$ (consistent, e.g., with the average gas density in the $z \sim 2$ protocluster studied by Hennawi et al. 2015). By substituting these values in Eqs. (15) and (16) of Gilli et al. (2017), we find that after $70 \mathrm{Myr}$, the bubble radius has expanded to $117 \mathrm{kpc}$, in excellent agreement with the measured size of component A of the diffuse X-ray emission $(\sim 120 \mathrm{kpc}$ radius).

We are also now in a position to compare the timescales of the star formation of galaxies $m 1-m 4$ with the time needed for the shock to cross them. On the one hand, we may expect that star formation is promoted by the compression of the cold gas in the galaxies as the shock approaches them. On the other hand, star formation may be quenched by removal and heating of their ISM (e.g., through ram-pressure stripping and thermal conduction, respectively; Vollmer et al. 2001; Tonnesen \& Bryan 2009; Vijayaraghavan \& Sarazin 2017) after the shock has crossed them and they are immersed within the hot $(T>5 \mathrm{keV})$ plasma of the bubble. In this scenario the time needed by the shock to cross the galaxies $m 1-m 4$ should be similar to the age of the burst of star formation producing most of the observed optical and UV emission of $m 1-m 4$. Based on the fit to the UV spectra of galaxies $m 1-m 4$ with stellar population synthesis models (see Sect. 4.2), we found significant episodes of star formation as young as a few megayears in all galaxies. Furthermore, in $m 1$ and in $m 4$ these recent bursts produce most of the optical and UV light. Using Eq. (18) of Gilli et al. (2017), we derived the expansion velocity of the hot gas bubble at $t=70 \mathrm{Myr}$, which is $v=1240 \mathrm{~km} \mathrm{~s}^{-1}$ and corresponds to $\sim 1.2 \mathrm{kpc} \mathrm{Myr}^{-1}$. Galaxies $m 1-m 4$ appear as compact systems in the HST and LBT/SOUL images. Based on the results obtained with SOUL on $m 3$ (and also on the additional candidate radio galaxy with $z_{\text {phot }} \approx 1.7$ ), a typical diameter of 4-5 kpc may be considered for these systems. The time needed by the shock to cross the galaxies is therefore comparable with the age and duration of recent bursts of star formation, which has to be expected if this is truly promoted by the compression of their ISM by the approaching shock front.

\footnotetext{
6 Note that $R_{0}$ is not $R(t=0)$; see Gilli et al. (2017).
}
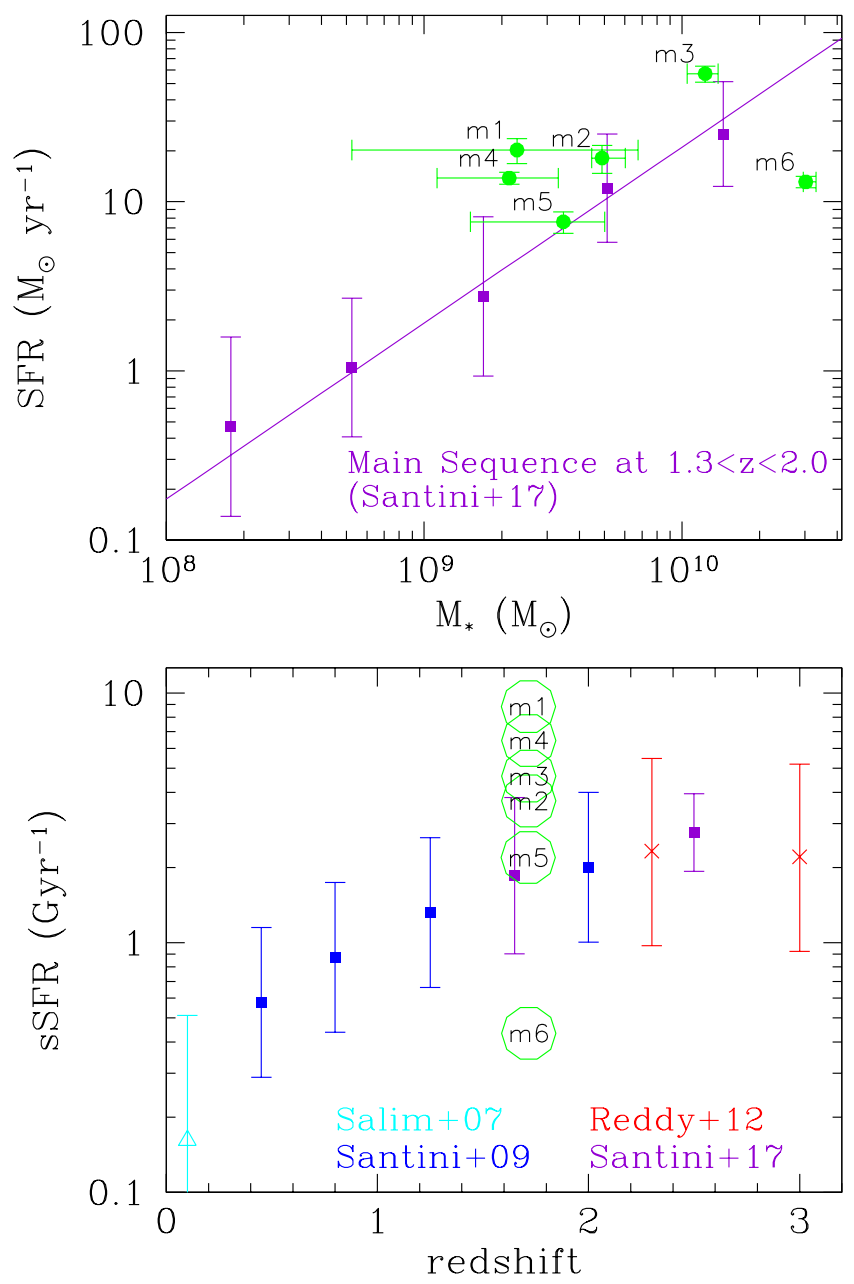

Fig. 13. Top panel: star formation rate vs. stellar mass for the overdensity galaxies $m 1-m 6$ (green circles). The violet squares and line show the main sequence of field galaxies at $1.3<z<2.0$ and its best-fit relation as derived by Santini et al. (2017). Bottom panel: specific star formation rate of the galaxies $m 1-m 6$ (green circles) plotted against the specific star formation rate vs. redshift relation derived for field galaxies with $\log \left(M_{*} / M_{\odot}\right)=9.5-10.0$. Literature data points are labeled.

To validate this scenario, we verified whether the sSFR, that is, the star formation rate per unit stellar mass, in the galaxies $m 1-m 4$ is effectively higher than that of galaxies $m 5$ and $m 6$ and than that of field galaxies of similar mass at the same redshift. In Fig. 13 (top panel) we show the SFR vs. stellar mass $M_{*}$ for $m 1-m 6$ derived in Sect. 4.2 compared with the average relation (the so-called main-sequence) derived by Santini et al. (2017) for galaxies at $z=1.3-2.0$ in the HST Frontier fields. Among the six MUSE galaxies in the overdensity $m 1-m 6$, the four at the bubble boundary, $m 1-m 4$, have SFRs higher than those expected in main-sequence galaxies, whereas $m 5$ and $m 6$ are on and below the main sequence, respectively. As shown in the bottom panel of the same figure, $m 1-m 4$ feature the highest sSFRs of the six MUSE galaxies at $z=1.7$. These rates are a factor of $2-5$ higher than the average sSFR of field galaxies of similar mass at $z=1.7$. Although we cannot make strong statements on the statistical significance of this result, we remark that it is consistent with the expectations of the proposed positive AGN feedback scenario. In the future, observations of UV rest-frame emission lines may be a powerful diagnostics to confirm the effectiveness of the incoming shock in promoting star formation in these galaxies by compressing and cooling their ISM. 
As a final remark, we note that the positive feedback scenario may also hold if the diffuse X-rays seen in component A are produced by IC-CMB rather than by shock-heated gas. In this case, a conservative lower limit to the total energy density $U_{\text {tot }}$, and hence to the total nonthermal pressure of the lobe $P_{\text {tot }}=U_{\text {tot }} / 3$, can be computed assuming that the system is in the state of minimum energy density $U_{\text {tot }}=(7 / 3) U_{B}$, where $U_{B}=B^{2} / 8 \pi$ is the magnetic energy density (this state is also often referred to as equipartition because the energies of the relativistic particles and magnetic field are approximately equal). By assuming $B=B_{\mathrm{eq}}=5 \mu \mathrm{G}$ (see Sect. 5.3) and the fiducial density and temperatures of the cold ambient medium discussed in this Section, we obtain $P_{\text {tot }} / P_{\text {cold }}>260$. This suggests again that the lobe is highly overpressurized and hence expands, compressing the ISM of the galaxies at its border and enhancing their star formation.

\section{Conclusions}

We have reported the discovery of a galaxy overdensity around an FRII radio galaxy at $z=1.69$ in the multiband survey around the $z=6.3$ QSO SDSS J1030+0524. This structure is likely still far from virialization phase and features significant evidence for positive AGN feedback produced by the radio galaxy. The field features deep multiwavelength coverage from the radio band to the X-rays. We analyzed and discussed observations with VLT/MUSE, LBT/LUCI, LBT/SOUL, VLA, and Chandra. Our main results are summarized below.

- We identified six overdensity members with MUSE and two members with LUCI. Their spectroscopic redshifts cover the range $z=1.687-1.699$. This structure extends for at least $800 \mathrm{kpc}$ on the plane of the sky (Fig. 1) and constitutes a significant (false-detection probability $<3.5 \times 10^{-7}$ ) overdensity of $\delta_{\mathrm{g}}=22$ in redshift space (Fig. 3). Based on the measured overdensity level and structure volume, we derive a lower limit to the total mass of the system of $1-2 \times 10^{13} M_{\odot}$. Most overdensity members are compact and blue galaxies that are forming stars at rates of $8-60 M_{\odot} \mathrm{yr}^{-1}$. We do not observe any strong galaxy morphological segregation or concentration around the FRII core. The structure is then populated by gas-rich galaxies and is still far from being virialized. It likely constitutes the progenitor of a local massive galaxy group or cluster caught during its main assembly phase.

- One of the two galaxies studied with LUCI is the host of the FRII, for which a redshift of $z=1.699$ was measured. The radio structure of the FRII extends for $\sim 600 \mathrm{kpc}$ across the sky. By combining VLA radio data at $1.4 \mathrm{GHz}$ with TGSS data at $150 \mathrm{MHz}$, we derived a kinetic power, velocity, and inclination angle of the FRII jet of $P_{\text {kin }}=6.3 \times 10^{45} \mathrm{erg} \mathrm{s}^{-1}$, $v=0.4-0.5 c$ and $\theta=70-80 \mathrm{deg}$, respectively.

- Based on a $500 \mathrm{ks}$ Chandra ACIS-I observation, we found that the FRII nucleus hosts a luminous QSO $\left(L_{2-10}=1.3 \times\right.$ $10^{44} \mathrm{erg} \mathrm{s}^{-1}$, intrinsic and rest-frame) that is obscured by Compton-thick absorption $\left(N_{\mathrm{H}}=1.5 \pm 0.6 \times 10^{24} \mathrm{~cm}^{-2}\right.$; Fig. 7). Under standard bolometric corrections the measured disk radiative power $\left(L_{\mathrm{rad}} \sim 4 \times 10^{45} \mathrm{erg} \mathrm{s}^{-1}\right)$ is similar to the jet kinetic power estimated from radio observations, as is commonly found in powerful jetted AGN.

- We found several regions of diffuse X-ray emission within the structure (see Figs. 8, 9, and 12). The most significant component (component A) extends for $\sim 240 \mathrm{kpc}$ in diameter around the eastern lobe of the FRII. We investigated the origin of this component and conclude that the most plausible explanation is thermal emission from an expanding bubble of gas that is shock-heated to a temperature of $T \approx 5 \mathrm{keV}$ by the FRII jet and carries an internal energy of $E_{\mathrm{th}} \sim 7 \times 10^{60} \mathrm{erg}$. We note that it is difficult to accurately measure the gas energy and temperature because of some possible IC-CMB contribution to the X-ray emission. However, the estimated values can be easily explained by the measured jet power and velocity if the system lifetime is $\sim 70 \mathrm{Myr}$, which is typical of FRIIs. By means of a simple model for the expansion of hot gas bubbles inflated by exponentially growing supermassive black holes, we verified that the measured radius of $\sim 120 \mathrm{kpc}$ of the diffuse X-ray component $\mathrm{A}$ is in excellent agreement with the expected radius given the estimated jet power and lifetime.

- Four $(m 1-m 4)$ out of the six MUSE star-forming galaxies in the overdensity are distributed in an arc-like shape at the edge of the main component of the diffuse X-ray emission. These four objects are concentrated within $200 \mathrm{kpc}$ in the plane of the sky and within $450 \mathrm{kpc}$ in radial separation. Three of them are even more concentrated, falling within $60 \mathrm{kpc}$ in both transverse and radial direction. The probability of observing four out of these six actively star-forming galaxies at the edge of the diffuse emission by chance is negligible. We then propose that star formation in these galaxies is promoted by the compression of their ISM by the approaching shock front, which would be remarkable evidence of positive AGN feedback on cosmological scales. The sSFRs of $m 1-m 4$ are the highest of the six MUSE overdensity members (see Fig. 13) and lie above the average sSFR of field galaxies with similar mass at $z=1.7$, in agreement with the proposed positive AGN feedback scenario. We emphasize that our conclusions about the feedback are robust because even when we assume that all of the diffuse X-ray emission is due to IC-CMB, star formation can still be promoted by the nonthermal pressure of the expanding lobe.

In the near future, several multiwavelength observational campaigns will bring further insight into the physics and structure of the overdensity. As an example, we recently observed the whole region around the FRII radio galaxy with a small ALMA mosaic to reveal a possible molecular gas reservoir around the FRII host and discover additional cluster galaxy members through the detection of the $\mathrm{CO}(2-1)$ emission line. In addition, we observed the field at $1.4 \mathrm{GHz}$ with the Jansky Very Large Array down to $\sim 1.8 \mu \mathrm{Jy} \mathrm{rms}$, that is, one dex deeper than what is currently available, and the newly allocated LOFAR observations will improve the angular resolution at $150 \mathrm{MHz}$ by a factor of three with respect to the existing TGSS data. The new radio datasets are expected to probe the morphology of the radio structure and reveal its interplay with the observed diffuse X-ray emission with an unprecedented level of detail for a distant large-scale structure.

Acknowledgements. We wish to thank the referee for a careful and critical review of the paper that helped us to present our results in a more balanced way. We acknowledge the support from the LBT-Italian Coordination Facility for the execution of observations, data distribution and reduction. The LBT is an international collaboration among institutions in the United States, Italy and Germany. LBT Corporation partners are the University of Arizona on behalf of the Arizona university system; Istituto Nazionale di Astrofisica, Italy; LBT Beteiligungsgesellschaft, Germany, representing the Max-Planck Society, the Astrophysical Institute Potsdam, and Heidelberg University; The Ohio State University, and The Research Corporation, on behalf of The University of Notre Dame, University of Minnesota and University of Virginia. The scientific results reported in this article are partly based on observations made by the Chandra X-ray Observatory. This work made use of data taken under the ESO program ID 095.A0714(A). We acknowledge useful discussions with L. Pozzetti and S. Ettori. We thank P. Santini for providing the literature data points of Fig. 13. We acknowledge financial contribution from the agreement ASI-INAF n. 2017-14-H.O. IP and QD acknowledge support from INAF under the PRIN SKA project FORECaST (1.05.01.88.03). 


\section{References}

Acker, A., Köppen, J., Samland, M., \& Stenholm, B. 1989, The Messenger, 58, 44

Annunziatella, M., Marchesini, D., Stefanon, M., et al. 2018, PASP, 130, 124501

Bacon, R., Accardo, M., Adjali, L., et al. 2010, in Ground-based and Airborne Instrumentation for Astronomy III, Proc. SPIE, 7735, 773508

Bacon, R., Vernet, J., Borisova, E., et al. 2014, The Messenger, 157, 13

Bacon, R., Conseil, S., Mary, D., et al. 2017, A\&A, 608, A1

Balestra, I., Tozzi, P., Ettori, S., et al. 2007, A\&A, 462, 429

Balmaverde, B., Gilli, R., Mignoli, M., et al. 2017, A\&A, 606, A23

Beckwith, S. V. W., Stiavelli, M., Koekemoer, A. M., et al. 2006, AJ, 132, 1729

Beers, T. C., Flynn, K., \& Gebhardt, K. 1990, AJ, 100, 32

Bertin, E., \& Arnouts, S. 1996, A\&AS, 117, 393

Bicknell, G. V., Sutherland, R. S., van Breugel, W. J. M., et al. 2000, ApJ, 540, 678

Blandford, R. D., \& Znajek, R. L. 1977, MNRAS, 179, 433

Bolzonella, M., Miralles, J.-M., \& Pelló, R. 2000, A\&A, 363, 476

Bolzonella, M., Kovač, K., Pozzetti, L., et al. 2010, A\&A, 524, A76

Bourne, M. A., Sijacki, D., \& Puchwein, E. 2019, MNRAS, 490, 343

Broos, P. S., Townsley, L. K., Feigelson, E. D., et al. 2010, ApJ, 714, 1582

Bruzual, G., \& Charlot, S. 2003, MNRAS, 344, 1000

Calzetti, D., Armus, L., Bohlin, R. C., et al. 2000, ApJ, 533, 682

Cash, W. 1979, ApJ, 228, 939

Chan, J. C. C., Beifiori, A., Saglia, R. P., et al. 2018, ApJ, 856, 8

Chiaberge, M., Capetti, A., Macchetto, F. D., et al. 2010, ApJ, 710, L107

Cid Fernandes, R., Mateus, A., Sodré, L., Stasińska, G., \& Gomes, J. M. 2005, MNRAS, 358, 363

Cid Fernandes, R., Stasińska, G., Mateus, A., \& Vale Asari, N. 2011, MNRAS, 413,1687

Cohen, M. H., Lister, M. L., Homan, D. C., et al. 2007, ApJ, 658, 232

Costa, T., Sijacki, D., \& Haehnelt, M. G. 2014, MNRAS, 444, 2355

Croft, S., van Breugel, W., de Vries, W., et al. 2006, ApJ, 647, 1040

Cucciati, O., Zamorani, G., Lemaux, B. C., et al. 2014, A\&A, 570, A16

Cucciati, O., Lemaux, B. C., Zamorani, G., et al. 2018, A\&A, 619, A49

Dey, A., van Breugel, W., Vacca, W. D., \& Antonucci, R. 1997, ApJ, 490, 698

Ehlert, K., Pfrommer, C., Weinberger, R., Pakmor, R., \& Springel, V. 2019, ApJ, 872, L8

Emonts, B. H. C., Lehnert, M. D., Dannerbauer, H., et al. 2018, MNRAS, 477, L60

Esposito, S., Riccardi, A., Pinna, E., et al. 2011, in Astronomical Adaptive Optics Systems and Applications IV, Proc SPIE, 8149, 814902

Fan, X., Narayanan, V. K., Lupton, R. H., et al. 2001, AJ, 122, 2833

Fragile, P. C., Anninos, P., Croft, S., Lacy, M., \& Witry, J. W. L. 2017, ApJ, 850, 171

Freeman, P. E., Kashyap, V., Rosner, R., \& Lamb, D. Q. 2002, ApJS, 138, 185

Garilli, B., Paioro, L., Scodeggio, M., et al. 2012, PASP, 124, 1232

Gawiser, E., van Dokkum, P. G., Herrera, D., et al. 2006, ApJS, 162, 1

Ghisellini, G., Haardt, F., Della Ceca, R., Volonteri, M., \& Sbarrato, T. 2013, MNRAS, 432, 2818

Ghisellini, G., Tavecchio, F., Maraschi, L., Celotti, A., \& Sbarrato, T. 2014, Nature, 515, 376

Gilli, R., Cimatti, A., Daddi, E., et al. 2003, ApJ, 592, 721

Gilli, R., Calura, F., D’Ercole, A., \& Norman, C. 2017, A\&A, 603, A69

Giovannini, G., Cotton, W. D., Feretti, L., Lara, L., \& Venturi, T. 2001, ApJ, 552, 508

Hardcastle, M. J., Evans, D. A., \& Croston, J. H. 2007, MNRAS, 376, 1849

Harris, D. E., \& Grindlay, J. E. 1979, MNRAS, 188, 25

Harwood, J. J., Hardcastle, M. J., Morganti, R., et al. 2017, MNRAS, 469, 639

Hayashi, M., Kodama, T., Kohno, K., et al. 2017, ApJ, 841, L21

Hennawi, J. F., Prochaska, J. X., Cantalupo, S., \& Arrigoni-Battaia, F. 2015, Science, 348, 779

Hillel, S., \& Soker, N. 2016, MNRAS, 455, 2139
Inami, H., Bacon, R., Brinchmann, J., et al. 2017, A\&A, 608, A2 Isobe, N., Seta, H., \& Tashiro, M. S. 2011, PASJ, 63, S947

Jolley, E. J. D., Kuncic, Z., Bicknell, G. V., \& Wagner, S. 2009, MNRAS, 400, 1521

Kennicutt, R. C., \& Evans, N. J. 2012, ARA\&A, 50, 531

Kim, S., Stiavelli, M., Trenti, M., et al. 2009, ApJ, 695, 809

Kotyla, J. P., Chiaberge, M., Baum, S., et al. 2016, ApJ, 826, 46

Kreckel, K., Groves, B., Bigiel, F., et al. 2017, ApJ, 834, 174

Lahav, O., Lilje, P. B., Primack, J. R., \& Rees, M. J. 1991, MNRAS, 251, 128

Lemaux, B. C., Gal, R. R., Lubin, L. M., et al. 2012, ApJ, 745, 106

Lin, L., Dickinson, M., Jian, H.-Y., et al. 2012, ApJ, 756, 71

Madau, P., \& Dickinson, M. 2014, ARA\&A, 52, 415

Marconi, A., Risaliti, G., Gilli, R., et al. 2004, MNRAS, 351, 169

Migliori, G., Grandi, P., Palumbo, G. G. C., Brunetti, G., \& Stanghellini, C. 2007, ApJ, 668, 203

Miley, G. 1980, ARA\&A, 18, 165

Miley, G., \& De Breuck, C. 2008, A\&ARv, 15, 67

Morselli, L., Mignoli, M., Gilli, R., et al. 2014, A\&A, 568, A1

Moster, B. P., Somerville, R. S., Newman, J. A., \& Rix, H.-W. 2011, ApJ, 731, 113

Mulchaey, J. S. 2000, ARA\&A, 38, 289

Murphy, K. D., \& Yaqoob, T. 2009, MNRAS, 397, 1549

Nanni, R., Gilli, R., Vignali, C., et al. 2018, A\&A, 614, A121

Nantais, J. B., Rettura, A., Lidman, C., et al. 2013, A\&A, 556, A112

Noble, A. G., McDonald, M., Muzzin, A., et al. 2017, ApJ, 842, L21

Noll, S., \& Pierini, D. 2005, A\&A, 444, 137

Nordon, R., Lutz, D., Saintonge, A., et al. 2013, ApJ, 762, 125

Overzier, R. A. 2016, A\&ARv, 24, 14

Overzier, R. A., Harris, D. E., Carilli, C. L., et al. 2005, A\&A, 433, 87

Pacholczyk, A. G. 1970, Radio Astrophysics. Nonthermal Processes in Galactic and Extragalactic Sources (San Francisco: Freeman)

Pentericci, L., Kurk, J. D., Röttgering, H. J. A., et al. 2000, A\&A, 361, L25

Petric, A. O., Carilli, C. L., Bertoldi, F., et al. 2003, AJ, 126, 15

Pinna, E., Esposito, S., Hinz, P., et al. 2016, in Adaptive Optics Systems V, SPIE Conf. Ser., 9909, 99093V

Planck Collaboration XIII. 2016, A\&A, 594, A13

Quadri, R., Marchesini, D., van Dokkum, P., et al. 2007, AJ, 134, 1103

Rosati, P., Borgani, S., \& Norman, C. 2002, ARA\&A, 40, 539

Salpeter, E. E. 1955, ApJ, 121, 161

Santini, P., Fontana, A., Castellano, M., et al. 2017, ApJ, 847, 76

Santos, J. S., Altieri, B., Valtchanov, I., et al. 2015, MNRAS, 447, L65

Shankar, F., Cavaliere, A., Cirasuolo, M., \& Maraschi, L. 2008, ApJ, 676, 131

Smail, I., Blundell, K. M., Lehmer, B. D., \& Alexander, D. M. 2012, ApJ, 760, 132

Smolčić, V., Novak, M., Delvecchio, I., et al. 2017, A\&A, 602, A6

Soto, K. T., Lilly, S. J., Bacon, R., Richard, J., \& Conseil, S. 2016, MNRAS, 458,3210

Steidel, C. C., Adelberger, K. L., Dickinson, M., et al. 1998, ApJ, 492, 428

Stiavelli, M., Djorgovski, S. G., Pavlovsky, C., et al. 2005, ApJ, 622, L1

Talia, M., Mignoli, M., Cimatti, A., et al. 2012, A\&A, 539, A61

Talia, M., Cimatti, A., Pozzetti, L., et al. 2015, A\&A, 582, A80

Tonnesen, S., \& Bryan, G. L. 2009, ApJ, 694, 789

Vijayaraghavan, R., \& Sarazin, C. 2017, ApJ, 848, 63

Vollmer, B., Cayatte, V., Balkowski, C., \& Duschl, W. J. 2001, ApJ, 561, 708

Weaver, R., McCray, R., Castor, J., Shapiro, P., \& Moore, R. 1977, ApJ, 218, 377

Webb, T. M. A., Lowenthal, J., Yun, M., et al. 2017, ApJ, 844, L17

Weilbacher, P. M., Streicher, O., Urrutia, T., et al. 2014, in Astronomical Data Analysis Software and Systems XXIII, eds. N. Manset, \& P. Forshay, ASP Conf. Ser., 485, 451

Willott, C. J., Rawlings, S., Blundell, K. M., \& Lacy, M. 1999, MNRAS, 309, 1017

Yaqoob, T. 1997, ApJ, 479, 184

Zirbel, E. L., \& Baum, S. A. 1995, ApJ, 448, 521 